REVIEW ARTICLE

\title{
Application of Raman spectroscopy to probe fundamental properties of two-dimensional materials
}

\author{
Xin Cong ${ }^{1,2}$, Xue-Lu Liu ${ }^{1}$, Miao-Ling $\operatorname{Lin}^{1}$ and Ping-Heng Tan $\mathbb{D}^{1,2,3 凶}$
}

Two-dimensional materials (2DMs), with remarkably electronic, optical, and mechanical properties, exhibit both high scientific interest and huge application potential. Raman spectroscopy has been proven to be a fast, convenient, and nondestructive technique to characterize the fundamental properties of 2DMs at both laboratory and mass-production scales. In this review, we discuss recent advances in application of Raman spectroscopy to 2DMs for probing their fundamental properties. First, we introduce Raman characterization on different types of 2DMs, phase transition triggered by defect, electrostatic doping and temperature, thickness-dependent intralayer and interlayer modes, and two-dimensional alloys with tunable compositions. The extensive capabilities of Raman spectroscopy in probing quantum phase transition are discussed, such as charge density wave and magnetic transition. Then, we discuss application of Raman spectroscopy to probe the moiré phonons, interfacial coupling and cross-dimensional electron-phonon coupling in van der Waals heterostructures (vdWHs). We hope that this review will be helpful to study the basic properties of 2DMs and vdWHs themselves and those present in the related devices by Raman spectroscopy.

npj 2D Materials and Applications (2020)4:13; https://doi.org/10.1038/s41699-020-0140-4

\section{INTRODUCTION}

Last century, the layered materials have been widely investigated, especially ion intercalated compounds, which promotes investigation of rechargeable batteries ${ }^{1,2}$. However, the single layer component of layered materials is unreachable, until graphene, a true two-dimensional materials (2DMs) with atomic thickness, is exfoliated by Novoselov et al. ${ }^{3}$. Graphene exhibits many extraordinary properties and its discovery stimulates a tremendous interest in two-dimensional materials (2DMs), with a wide range from insulator, topological insulators, semiconductor, semimetal, and metal to superconductors ${ }^{4-7}$. The recent advance in synthesis of diverse 2DMs provides opportunities and versatile platform for investigation of phenomena for fundamental science, such as massless Dirac fermions, superconductors, ferromagnetics, half-integer quantum Hall effect, and potential application in highend electronic, spintronics, optoelectronics, energy harvesting, and flexible electronics ${ }^{6,7}$. Due to ultrathin thickness, the properties of 2DMs, such as band structures, lattice vibrations, and electron-phonon interaction, are sensitive to preparation methods, sizes, substrate, compositions, thickness, doping, defects, vacancies, strain, crystal phases, etc ${ }^{8}$. Furthermore, recent research studies have advanced to investigate unusual properties and exceptional device performance of vertical van der Waals heterostructure (vdWHs) based on vertical stacking of 2DMs layer by layer in precise sequence by vdW interaction?. The vdWHs, without any restriction of lattice matching and fabrication compatibility, combine advantages of different 2DMs and offer huge opportunities for the design of new functionalities ${ }^{9,10}$. A convenient and in situ characterization technology is necessary for identifying various fundamental properties of 2DMs and vdWHs.

Among the variety of characterization methods ${ }^{6}$, Raman spectroscopy, one of a fast and nondestructive characterization method with high spatial and spectral resolution, stands out and is applicable at both laboratory and mass-production scales ${ }^{8,11,12}$. In general, Raman peaks of lattice vibrations (i.e., phonons) in 2DMs exhibit several prominent features, including line shape, peak position (Pos), full width at half maximum (FWHM), and intensity (I), which contain useful information to characterize physical and chemical properties of $2 \mathrm{DMs}$, such as quantum interference, phonon frequency, decay rate of intermediate state and Raman process, electron-phonon coupling, electronic states, etc ${ }^{8}$. According to the atomic displacement of lattice vibrations, there are two kinds of Raman modes in 2DMs, intralayer and interlayer modes, stemming from intralayer chemical bonds and layer-layer vdW interaction, respectively ${ }^{13}$. The features of intralayer Raman modes provide information of compositions and structural phase. Their response to external perturbation provides opportunities to investigate fundamental properties of $2 \mathrm{DMs}$, such as temperature dependence for phonon anharmonicity, electron-phonon coupling (EPC) and thermal expansion ${ }^{14,15}$, electrostatic doping dependence for $E P C^{16,17}$, strain dependence for elastic constant ${ }^{18,19}$, defect concentration dependence for phonon confinement effect ${ }^{20-23}$, magnetic field dependence for Fermi velocity and many-body effect ${ }^{24,25}$, and excitation energy $\left(E_{\text {ex }}\right)$ dependence for band structure, double resonance Raman process ${ }^{11,26}$, interlayer $\mathrm{EPC}^{27}$, and phonon dispersion ${ }^{28-30}$. The interlayer Raman modes involve layer-layer vibration where each layer can be treated as a whole unit, which is known as linear chain model (LCM) for Raman spectroscopy ${ }^{13,31,32}$. Based on the low-frequency Raman technique developed by Tan et al. ${ }^{13}$, the interlayer Raman modes of 2DMs can be observed easily. This stimulates huge interest to investigate the interlayer coupling in 2DMs and related vdWHs, which is highly sensitive to the thickness and stacking order of 2DM flakes ${ }^{33}$. LCM can also be extended to vdWHs to investigate interfacial coupling ${ }^{34}$ and cross-dimensional EPC $^{35}$. Furthermore, moiré pattern induced by periodic potential in vdWHs results in that non-center intralayer phonons of the constituents are folded back to the center of Brillouin zone (BZ) in

\footnotetext{
${ }^{1}$ State Key Laboratory of Superlattices and Microstructures, Institute of Semiconductors, Chinese Academy of Sciences, 100083 Beijing, China. ${ }^{2}$ Center of Materials Science and Optoelectronics Engineering \& CAS Center of Excellence in Topological Quantum Computation, University of Chinese Academy of Sciences, 100049 Beijing, China. ${ }^{3}$ Beijing Academy of Quantum Information Science, 100193 Beijing, China. ${ }^{凶}$ email: phtan@semi.ac.cn
} 
vdWHs and become Raman active, such as $R$ and $R^{\prime}$ modes in twisted multilayer graphene (tMLG) $)^{31}$ and moiré phonon in twisted bilayer $\mathrm{MoS}_{2}(\mathrm{t} 2 \mathrm{LM})^{30}$. All of those Raman features can be employed to probe the fundamental properties of 2DMs, including thickness, structural phase, doping level, elastic properties, etc ${ }^{8}$. Raman peaks originating from other excitations, such as correlated electron, spin and charge density, can also reveal intricate quantum phenomena in 2DMs, including superconductivity, spin dynamics, magnetic phase transition, and charge density wave (CDW) phase transition ${ }^{5,36-38}$.

Here, we systemically give an up-to-date overview of recent developments in application of Raman spectroscopy to characterize the fundamental properties of 2DMs and related vdWHs. First, we introduce Raman spectra of several types of 2DMs with different compounds, phase, thickness and tunable compositions, where composition, phase transition and thickness in 2DMs can be revealed and determined by the spectral features. And we take $\mathrm{MoTe}_{2}$ as an example to introduce layer-dependent interlayer and intralayer modes in 2DMs, which can be described by LCM and van der Waals model, respectively. Then, we present application of Raman spectroscopy to investigate intriguing quantum phenomena of 2DMs, such as quantum spin liquid and CDW states. In twodimensional quantum magnet $\mathrm{RuCl}_{3}$, the excitation of quantum spin liquid and relation between magnetism and structural phase reveal its magnetic phase transition. The modulation of charge density with coupling to phonons also provides opportunities for investigating CDW phase transition in related 2DMs. Furthermore, we discuss interlayer and intralayer Raman modes in vdWHs, including twisted bilayer $\mathrm{MoS}_{2}$ (t2LM), graphene/MoS 2 , and $\mathrm{WS}_{2}$ / $\mathrm{hBN}$ vdWHs. The moiré periodic potentials in t2LM make twist angle $(\theta)$ dependent moiré phonons be observed, which can be used to probe the phonon dispersion curve of monolayer $\mathrm{MoS}_{2}$. We take graphene/MoS 2 and $\mathrm{WS}_{2} / \mathrm{hBN}$ vdWHs as examples to demonstrate how to probe the interfacial coupling in vdWHs by their LB modes. Furthermore, the excitation-dependent intensity of the LB modes in $\mathrm{WS}_{2} / \mathrm{hBN}$ vdWHs reveals cross-dimensional
EPC. Finally, we conclude the review with the outlook for the further investigation on 2DMs by Raman spectroscopy.

\section{RAMAN CHARACTERIZATION OF 2DMS}

Raman spectra of different types of 2DMs

According to the involved elements, library of 2DMs can be categorized into several types, such as graphene family, twodimensional dichalcogenides and oxides ${ }^{10}$. The various 2DMs with wide spectrum can be insulator, topological insulator, semiconductor, semi-metal, conductor, or superconductor. Their Raman spectra are discrepant in position, width, and intensity of Raman peaks. Here, we take hexagonal boron nitride ( $\mathrm{hBN}$, insulator), $\mathrm{Bi}_{2} \mathrm{Se}_{3}$ (topological insulators), hexagonal $\mathrm{MoS}_{2}\left(2 \mathrm{H}-\mathrm{MoS}_{2}\right.$, in-plane isotropic semiconductors), black phphosphorus (BP, in-plane anisotropic semiconductors), graphene (semi-metals), and $\mathrm{MoCl}_{3}$ (magnetism) as examples to introduce Raman spectra of various 2DMs, as presented in Fig. 1.

In principle, first-order Raman peaks originate from lattice vibrations at the BZ center, which can be classified based on the irreducible presentation of symmetry group of the crystal. For example, the symmetry of monolayer graphene (1LG) is $D_{6 \mathrm{~h}}$, and the lattice vibrations of $1 \mathrm{LG}$ can be expressed as: $\Gamma_{1 \mathrm{LG}}=A_{2 \mathrm{u}}+B_{2 \mathrm{~g}}$ $+E_{1 \mathrm{u}}+E_{2 \mathrm{~g}}$. The three acoustic modes and three optical modes in $1 \mathrm{LG}$ correspond to one $A_{2 \mathrm{u}}$ mode and one $E_{1 \mathrm{u}}$ (double degenerate) mode, a doubly degenerate in-plane mode $\left(E_{2 g}\right)$, and one out-of-plane mode $\left(B_{2 g}\right)$, respectively ${ }^{11}$. Figure 1a shows typical Raman spectra of pristine $1 \mathrm{LG}$, with two prominent Raman peaks, the so-called G-mode at around $1580 \mathrm{~cm}^{-1}$ and 2D mode at around $2700 \mathrm{~cm}^{-1}$. The G-band is associated with the doubly degenerate (longitudinal optical (LO) and in-plane transverse optical (TO)) phonon mode ( $E_{2 g}$ symmetry) at the BZ center and is a characteristic peak of graphene-related materials ${ }^{11}$. The $2 D$ peak arises from the overtone of TO phonon around $\mathrm{K}$ point and is activated by double resonance Raman scattering, where its lineshape is sensitive to electronic band structure. Hexagonal boron nitride ( $\mathrm{hBN}$ ) exhibits similar crystal structure to graphene,
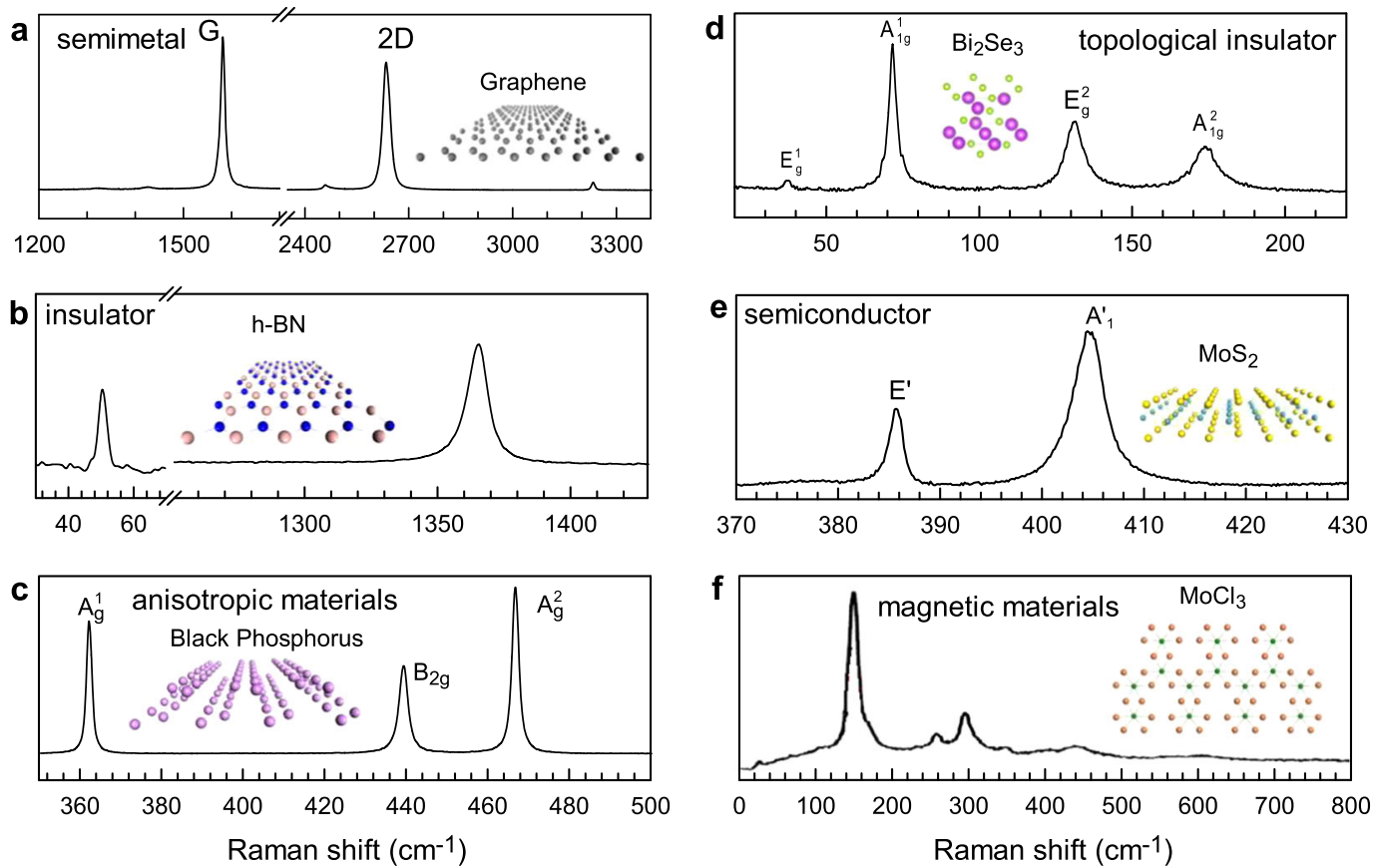

Fig. 1 Raman spectroscopy of representative 2DMs. a Graphene (semimetal), b h-BN (insulator), c BP (anisotropic semiconductor), d Bi ${ }_{2} \mathrm{Se}_{3}$ (topological insulator), e $\mathrm{MoS}_{2}$ (semiconductor), $\mathbf{f ~} \mathrm{MoCl}_{3}$ (magnetic materials). The inserts presents their corresponding crystal structures. All Raman spectra are obtained at room temperature. Reprinted figure $\mathbf{f}$ with permission from ref. ${ }^{82}$. Copyright (2017) by the American Physical Society ${ }^{39}$. 
in which two carbon atoms in the unit cell are replaced by $\mathrm{B}$ and $\mathrm{N}$ atoms, and its lattice vibrations are also similar to that of graphene with different frequencies, where $E_{2 g}$ mode in $\mathrm{hBN}$ lies at $\sim 1364.7 \mathrm{~cm}^{-1}$. In general, crystal with same symmetry but different atoms exhibit similar Raman peaks with different peak positions, which is summarized in a review article ${ }^{39}$. For 2DMs with distinct crystal structure, their lattice vibrations are distinct, where $\mathrm{BP}, \mathrm{MoS}_{2}, \mathrm{Bi}_{2} \mathrm{Se}_{3}$, and $\mathrm{MoCl}_{3}$ have $D_{2 \mathrm{~h}}, D_{3 \mathrm{~h}}, D_{3 \mathrm{~h}}$, and $C_{2 \mathrm{~h}}$ symmetry, respectively. Their Raman spectra exhibit distinguishable spectral features from each other, as presented in Fig. 1.

We note that Raman spectra in Fig. 1 is obtained at room temperature. In principle, Raman features, such as position, FWHM, and intensity of Raman peaks, are sensitive to temperature, which continuously changes with temperature varying. Raman spectra obtained at different temperature are different to each other, which can provide valuable information. The FWHM and peak position as a function of temperature can reveal contributions of phonon anharmonicity and electron-phonon coupling (phonons decaying into lower-energy phonons and creating an electronhole pair, respectively), and contributions of phonon anharmonicity and negative thermal expansion, respectively. Especially, graphene and graphite have been reported with negative thermal expansion, significant phonon anharmonicity and electron-phonon coupling ${ }^{14,15,40}$. Meanwhile, the doping exhibits significant influence on Raman features and temperaturedependent Raman spectra of graphene, and graphite can be employed to reveal intrinsic information. Furthermore, under doping, temperature or other external perturbation, drastic changes of Raman features might evidence phase transition, which is discussed latter.
Structural phase transition in transition metal dichalcogenides (TMDs)

2DMs can exhibit multiple structure phases with distinct symmetries and physical properties by combining involved atoms with different coordinations. Here, we take TMDs as examples to introduce diverse structural phases and their corresponding properties. Figure 2a summaries possible lattice structures of TMDs involved group VI transition metal (Mo, W). The unit layer of TMDs is formed by three atomic planes (chalcogen-metalchalcogen), and stacking sequence determines their structural phase, including $2 \mathrm{H}$ and $1 \mathrm{~T}$ or $1 \mathrm{~T}^{\prime}$ phases in monolayer TMDs, where $1 \mathrm{~T}^{\prime}$ indicates distorted $1 \mathrm{~T}$ phase. The $2 \mathrm{H}$ and $1 \mathrm{~T}$ phases are described by a hexagonal $\left(D_{3 h}\right)$ and tetragonal $\left(D_{3 d}\right)$ symmetry, with the chalcogen atoms vertically aligned and shifted to the others along $c$-axis, respectively. For $1 \mathrm{~T}^{\prime}$ phase of bulk TMDs, monoclinic and orthorhombic stacking are referred as $\mathrm{T}_{\text {mo }}^{\prime}$ and $\mathrm{T}^{\prime}$ or phases, respectively ${ }^{41}$. Structural phases in monolayer TMDs correlate with electrons in the $d$ orbitals ${ }^{42}$. For $2 \mathrm{H}$ and $1 \mathrm{~T}$ phases of TMDs, the $d$ orbital splits into $3\left(d_{z^{2}}, d_{x^{2}-y^{2}, x y}\right.$ and $\left.d_{x y, y z}\right)$ and 2 $\left(d_{x y, y z, z x}\right.$ and $\left.d_{x^{2}-y^{2}, z^{2}}\right)$ degenerate states, respectively, resulting in diverse electronic properties of $2 \mathrm{H}$ and $1 \mathrm{~T}$ phases of TMDs ${ }^{5,43}$. In general, the $2 \mathrm{H}$ phase is thermodynamically stable and semiconducting, while the 1T phase is metastable and metallic. Structure transition between different phases with distinct properties can lead to some intriguing applications, such as electronic and energy homojunction devices. For example, highquality two-dimensional device with an ohmic contact can be achieved by the local phase transition from $2 \mathrm{H}$ to $1 \mathrm{~T}$ phase ${ }^{44}$. Furthermore, structural phase transitions can interrelate with quantum states, such as quantum spin hall insulator and various superconducting states ${ }^{43}$.
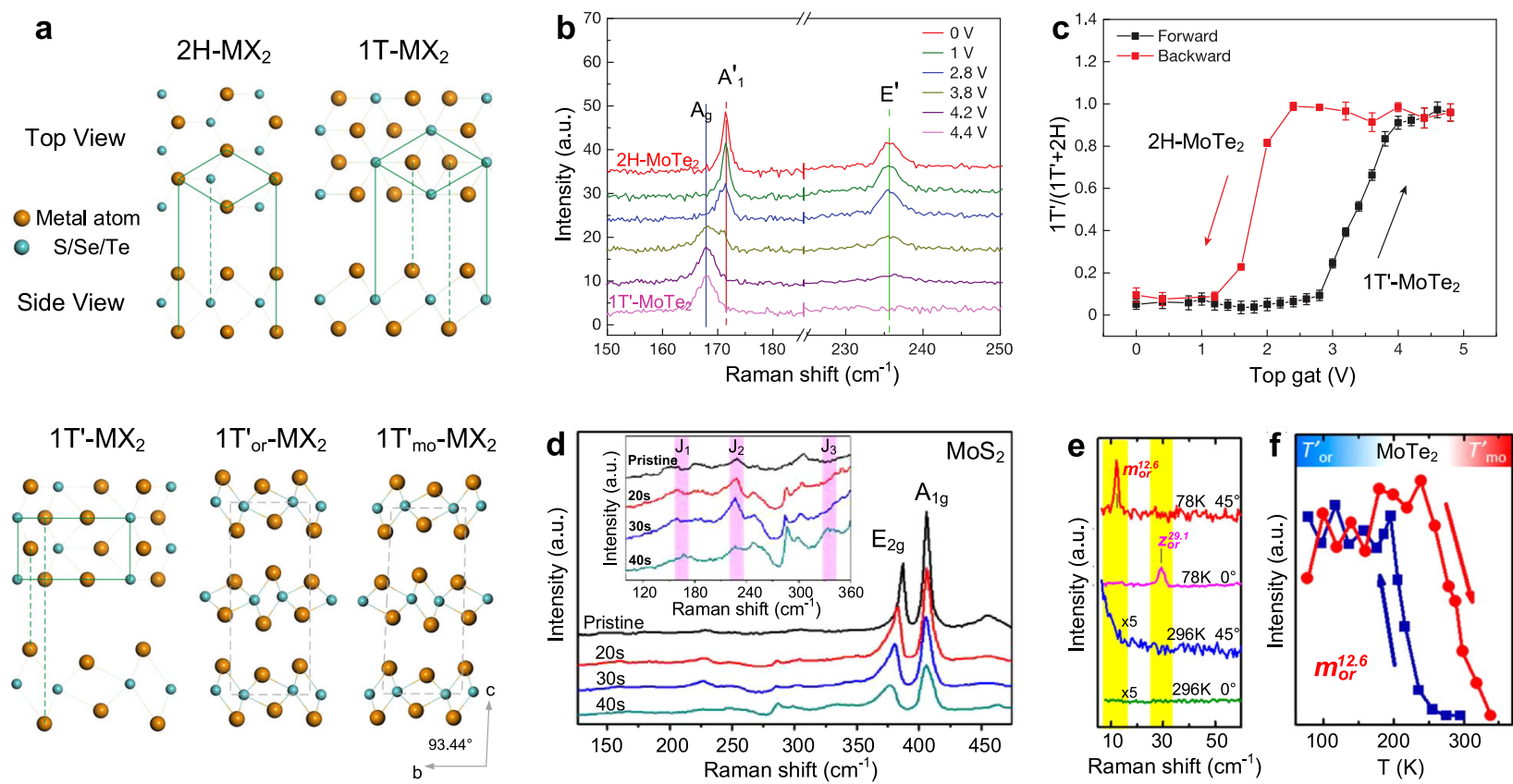

Fig. 2 Diverse structure of TMDs and phase transition between them. a TMDs crystal structure of $2 \mathrm{H}, 1 \mathrm{~T}, 1 \mathrm{~T}^{\prime}, 1 \mathrm{~T}^{\prime}{ }_{\mathrm{or}}$, and $1 \mathrm{~T}_{\mathrm{mo}}^{\prime}$ phases. b Representative Raman spectra before, during and after transition from the $2 \mathrm{H}$ to the $1 \mathrm{~T}^{\prime}$ phase of MoTe $\mathrm{T}_{2}$, as the bias changes from 0 to $4.4 \mathrm{~V}$. The characteristic Raman modes, $A_{1}^{\prime}$ and $E^{\prime}$ of $2 \mathrm{H}$ phase, and $\mathrm{A}_{\mathrm{g}}$ mode of $1 \mathrm{~T}^{\prime}$ phase are shown by red, green, and blue dashed lines, respectively. c Gate-dependent Raman intensity ratios, $F=1 \mathrm{~T}^{\prime}\left(A_{\mathrm{g}}\right) /\left[2 \mathrm{H}\left(A_{1}^{\prime}\right)+1 \mathrm{~T}^{\prime}\left(A_{\mathrm{g}}\right)\right]$ (y-axis). The black and red curves show increasing and decreasing gate voltage, respectively. $\mathbf{b}$, $\mathbf{c}$ Used with permission from Springer Nature ${ }^{45}$. $\mathbf{d}$ Raman spectra of pristine $2 \mathrm{H}-\mathrm{MoS}_{2}$ and plasmatreated $\mathrm{MoS}_{2}$, and new peaks induced by plasma treatment indicate a mixture of $1 \mathrm{~T}$ and $2 \mathrm{H}$ phase MoS 2 . The insert shows enlarged Raman spectra of $1 \mathrm{~T}^{-\mathrm{MoS}_{2}}$. Image d adapted with permission from Zhu et al. ${ }^{46}$ copyright [2017] American Chemical Society ${ }^{47}$. e The Raman spectra of $\mathrm{T}^{\prime}-\mathrm{MoTe}_{2}$ at 78 and $296 \mathrm{~K}$ obtained with $\theta=45^{\circ}$ and $0^{\circ}$ and vertically polarized scattering photons, where $\theta$ is the angle between the polarization of the incident laser light and the crystal $a$-axis. The yellow bands highlight appearance of new Raman peaks in $\mathrm{T}_{\text {or }}^{\prime}$ phase, comparing with $\mathrm{T}_{\mathrm{mo}}^{\prime}$ phase. f Temperature-dependent intensity of the $m_{\mathrm{or}}^{12.6}$ mode during cooling (dark blue) and warming (red) process. Images e, $\mathbf{f}$ adapted with permission from Chen et al. ${ }^{41}$. 
The transition between diverse structural phases can be driven through modifying electron filling of $d$ orbital. The electron injecting into $2 \mathrm{H}$ phase, such as electrostatic doping (Fig. 2b) ${ }^{45}$ and alkali metal interaction ${ }^{44}$, would increase electron density of the $d$ orbital and modulate filling of $d$ orbital, which further results in destabilization of the pristine $2 \mathrm{H}$ phase and phase transition to the metallic $1 \mathrm{~T}^{\prime}$ phase. The diverse structural phases of TMDs exhibit different symmetries with distinct Raman features. And Raman spectroscopy can provide a direct insight into the evolution process of phase transition. For example, Raman spectra of $2 \mathrm{H}-\mathrm{MoTe}_{2}$ with $D_{3 \mathrm{~h}}$ symmetry exhibit two prominent Raman features at $\sim 171.5 \mathrm{~cm}^{-1}\left(A^{\prime}\right)$ and $\sim 236 \mathrm{~cm}^{-1}\left(E^{\prime}\right)$, while that of $1 \mathrm{~T}$ '-MoTe 2 with $D_{3 d}$ symmetry exhibits obviously different Raman features with $A_{\mathrm{g}}$ mode at $\sim 167.5 \mathrm{~cm}^{-1}$, and the vanishing of $A_{1}^{\prime}$ and $E^{\prime}$ modes and rising of $A_{\mathrm{g}}$ mode indicate phase transition of $\mathrm{MoTe}_{2}$ from $2 \mathrm{H}$ to $1 \mathrm{~T}^{\prime}$ as increasing electrostatic bias (shown in Fig. $2 \mathrm{C}, \mathrm{d}$ ). The transition between $1 \mathrm{~T}$ and $2 \mathrm{H}$ phases can also be driven through modifying electron filling of $d$ orbital by vacancy defect, electron beam, plasma or laser beam irradiation ${ }^{42}$. As shown in Fig. $2 \mathrm{~d}$, three Raman peaks of $1 \mathrm{~T}-\operatorname{MoS}_{2}\left(J_{1}\left(\sim 167 \mathrm{~cm}^{-1}\right)\right.$, $\left.J_{2}\left(\sim 227 \mathrm{~cm}^{-1}\right), J_{3}\left(\sim 334 \mathrm{~cm}^{-1}\right)\right)$ appears in the Raman spectra of plasma-treated $2 \mathrm{H}-\mathrm{MoS}_{2}$, indicating presence of phase transition and mixture phases of $\mathrm{MoS}_{2}{ }^{46}$. Additionally, phase transition can be achieved by varying temperature. From room temperature to low temperature, $\mathrm{MoTe}_{2}$ with monoclinic stacking $\left(1 \mathrm{~T}_{\mathrm{mo}}^{\prime}\right)$ can transform to an orthorhombic phase $\left(1 \mathrm{~T}_{\mathrm{or}}^{\prime}\right)$ with symmetry breaking, resulting in different Raman-active modes corresponding to different lattice symmetries. Figure $2 \mathrm{e}, \mathrm{f}$ present Raman modes with different polarization configurations as a function of temperature, indicating phase transition and symmetry change. The transformation of phonon modes and the corresponding hysteresis loop in Fig. $2 \mathrm{~d}, \mathrm{f}$ indicate a clear reversible phase transition under gate control and temperature, respectively. All of the results indicate that Raman spectroscopy is a convenient method for revealing the competition, coexistence and cooperation of different structural phases.

\section{Thickness-dependent Raman modes}

Distinct from monolayer 2DMs, multilayer 2DMs stacked by monolayer constituents exhibit not only the intralayer vibrations, but also the interlayer vibrations, due to restoring force from vdW interlayer interaction ${ }^{47}$. The corresponding interlayer modes in a multilayer 2DM show a significant dependence on its thickness. The peak position of the intralayer modes, such as $E_{2 g}^{1}$ and $A_{1 g}$ modes in $2 \mathrm{H}-\mathrm{MoS}_{2}$ and $2 \mathrm{H}-\mathrm{MoTe}_{2}$, also exhibit distinct tendency with thickness from $1 \mathrm{~L}$ to bulk, which can be employed to identify the number of layers of 2DM flakes ${ }^{48}$. The thickness-dependent peak position of the interlayer modes and Davydov splitting of the intralayer modes provide a direct route to characterize the interlayer coupling in 2DMs.

Figure 3 presents thickness-dependent interlayer and intralayer modes of $2 \mathrm{H}-\mathrm{MoTe}_{2}$. According to the Raman selection rule, the LB and shear (S) modes can be distinguished by polarized Raman measurement, where the LB mode cannot be detected under the cross-polarized backscattering geometry ${ }^{48}$, as shown in Fig. $3 a$. The frequencies of interlayer S and LB modes as a function of thickness can be well reproduced by LCM (Fig. 3e) as follows: ${ }^{13}$

$$
\begin{aligned}
& \omega\left(\mathrm{S}_{\mathrm{N}, \mathrm{N}-\mathrm{i}}\right)=\frac{1}{\pi c} \sqrt{a^{\|} / \mu} \sin (i \pi / 2 N) \\
& \omega\left(\mathrm{LB}_{\mathrm{N}, \mathrm{N}-\mathrm{i}}\right)=\frac{1}{\pi c} \sqrt{a^{\perp} / \mu} \sin (i \pi / 2 N)
\end{aligned},
$$

where $i=1,2, \ldots, N-1$. For $N$-layer isotropic 2DMs, there are $N-$ 1 pairs of $S$ and $L B$ modes, respectively, denoted as $S_{N, N-i}$ and $L B_{N}$, $\mathrm{N}-\mathrm{i}, a^{\|}(\mathrm{Te})$ and $a^{\perp}(\mathrm{Te})$ are the nearest-neighbor force constants per unit area for the $\mathrm{S}$ and LB modes of $\mathrm{MoTe}_{2}$, respectively, and $\mu$ is the monolayer mass per unit area. As shown in Fig. 3e, the results of LCM are in excellent agreement with frequency of S and LB mode as a function of thickness with $a^{\|}(T e)=42.5 \times 10^{18} \mathrm{~N} \mathrm{~m}^{-3}$ and $a^{\perp}(T e)=91.2 \times 10^{18} \mathrm{Nm}^{-349}$. And the LCM can also be applied to describes shear and LB modes of 2DMs, such as as $\mathrm{TMDs}^{48}$, black phosphorus ${ }^{47}$, twist multilayer graphene ${ }^{31,32}$, $\mathrm{h}-\mathrm{BN}^{50}$.

The interlayer coupling can result in Davydov splitting of intralayer modes ${ }^{51-53}$, where the $A_{1}^{\prime} / A_{1 g}^{2}$ mode splits into several peaks with increasing thickness from $1-6 \mathrm{~L}$. Figure $3 f$ presents lattice vibrations of the $L B$ and $A s_{1} / A_{1 g}^{2}$ modes. Song et al. ${ }^{49}$ proposed a vdW model to estimate Davydov splitting of the intralayer $A_{1}^{\prime} / A_{1 \mathrm{~g}}^{2}$ modes. The frequency $\left(\omega_{\mathrm{cj}}\right)$ of each coupled Davydov component and the corresponding coupling frequency $\left(\Delta \omega_{\mathrm{j}}=\omega\left(\mathrm{LB}_{4 \mathrm{j}}\right)\right)$ have a relation of $\omega_{\mathrm{cj}}^{2}-\Delta \omega_{\mathrm{j}}^{2}=\omega_{0}^{2}(j=1,2,3)$, where $\omega_{0}$ is the frequency of the isolated entity. The vdW model reveals the effect of interlayer interaction on the intralayer lattice vibration. The model can also be extended to the intralayer Raman modes in other multilayer $\mathrm{TMDs}^{54}$.

\section{Alloy 2DMs}

Alloying two or more 2DMs with similar properties can obtain a new 2DM, so-called two-dimensional alloy, with tunable properties (e.g., tunable band gap) in a wide range. Varying composition of two-dimensional alloy enables us to achieve almost arbitrary control of properties at atomic scale. TMD-based Alloys with tunable band gaps have promising applications in nanoelectronics and optoelectronics ${ }^{55}$. The structure characterization of alloys is of primary importance for fundamental science and applications, which can be achieved by Raman spectroscopy with frequency shift and broadening of Raman peaks ${ }^{56,57}$.

Here, as an example, we discuss the evolution of Raman spectra of two-dimensional alloys involving $\mathrm{MoS}_{2}$ and $\mathrm{WS}_{2}$, i.e., $\mathrm{Mo}_{1-\mathrm{x}} \mathrm{W}_{\mathrm{x}} \mathrm{S}_{2}$. Figure 4 presents Raman spectra of $\mathrm{Mo}_{1-\mathrm{x}} \mathrm{W}_{\mathrm{x}} \mathrm{S}_{2}$ as a function of composition, where $x=0$ and 1 indicate pure $M_{0} S_{2}$ and $\mathrm{WS}_{2}$, respectively. According to symmetry analysis, two accessible first-order Raman active modes, $E^{\prime}$ and $A_{1}^{\prime}$ modes, can be observed in Raman spectra of monolayer $\operatorname{MoS}_{2}$ ( 385 and $\left.\sim 404 \mathrm{~cm}^{-1}\right)$ and $W_{2}\left(\sim 357 \text { and } \sim 419 \mathrm{~cm}^{-1}\right)^{48}$. The $E^{\prime}$ and $A_{1}^{\prime}$ modes, respectively, associate in-plane displacement of transition metal and $\mathrm{S}$ atom, and out-of-plane vibration involving $\mathrm{S}$ atoms, as shown in the insert of Fig. 4b. As increasing composition of $\mathrm{W}$ atom, two phonon branches associated to $E^{\prime}$ mode are observed in alloys, where the relative intensity of $\mathrm{WS}_{2}$-like mode increases, and that of $\mathrm{MoS}_{2}$-like $E^{\prime}$ mode exhibit contrast behavior. For the $A_{1}^{\prime}$ mode, its frequency shifts continuously as varying $\mathrm{W}$ composition. Figure $4 \mathrm{~b}$ presents frequency of $E^{\prime}$ and $A_{1}^{\prime}\left(E_{2 g}^{1}\right.$ and $A_{1 \mathrm{~g}}$ for bulk) modes as a function of the $\mathrm{W}$ composition. To comprehend their frequency shifts, modified random-element-isodisplacement (MREI) model is proposed, which involves interaction of S-Mo and $\mathrm{S}-\mathrm{W}$ along vertical (for $A_{1}^{\prime}$ mode) and horizontal (for $E^{\prime}$ mode) direction and Mo-W along horizontal direction (for $E^{\prime}$ mode) ${ }^{57}$. The experimental data can be well fitted by the MREI model, as shown in Fig. 4b.

As shown in Fig. 4a, the broadening of Raman peaks results from disorder. However, the disorder effect in multilayer alloys is absent in its interlayer ( $\mathrm{S}$ and LB) modes, which can also be well described by $\mathrm{LCM}^{58}$. The layer-dependent interlayer modes insensitive to in-plane disorder offer a robust and substrate-free approach for layer-number identification of two-dimensional alloys. This systematic investigation promotes the spectroscopic determination of composition, alloy degree and number of layers for various two-dimensional alloys.

\section{QUANTUM PHASE TRANSITION PROBED BY RAMAN SPECTROSCOPY}

Recently, intriguing quantum phenomena, such as CDW, intrinsic magnetism and superconductor, have been observed in 2DMs 

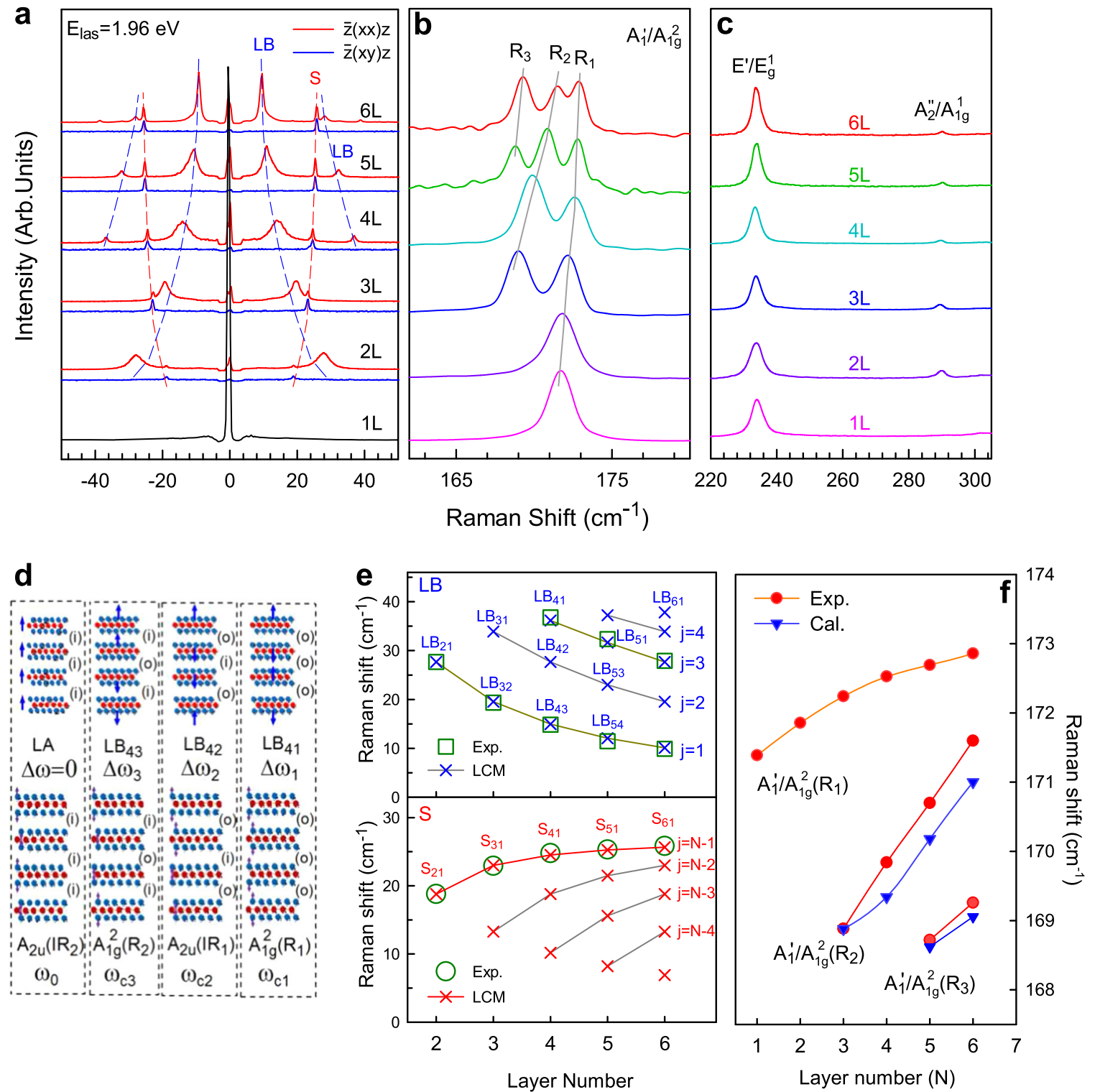

Fig. 3 Thickness-dependent Raman modes. a Raman spectra of 1-6 L MoTe 2 in range of shear (S) and LB modes, excited by 1.96 eV, under the parallel (red solid line) and perpendicular (blue solid line) polarization configuration. Blue and red dashed lines are guides for the eye for LB and shear modes, respectively. b, c Layer-dependent Raman spectra of 1-6 $\mathrm{L} \mathrm{MoTe}_{2}$ in high-frequency region excited by $1.96 \mathrm{eV}$. d Schematic of vdW model for atomic displacements of one LA mode, three LB modes and four Davydov components, in $4 \mathrm{~L}$ MoTe 2 . e The experimental frequencies and calculated ones of the LB (top) and S (bottom) modes based on LCM. f Comparison between experiment frequency (Exp.) and calculated (Cal.) frequency of each Davydov component in 3-6 L MoTe 2 based on the vdW model. Reprinted figures b-f with permission from Song et al. ${ }^{49}$.

and exhibit dimension dependence ${ }^{42,59-62}$. The quantum phase results from diverse interactions in 2DMs, such as strong electron correlation and electron-phonon interaction. Phase transition provides means to effectively modify their quantum states and related properties. Here, we discussed magnetism and CDW phase transition in 2DMs probed by Raman spectroscopy.

\section{CDW in 2DMs}

In 1955, Peierls pointed out that a one-dimensional (1D) metal with electrons and lattice interaction is not stable and would condense into CDW ground states at low temperature ${ }^{63}$. CDW in solids is periodic modulations of charge density at the Fermi energy, which is accompanied with bandgap opening, resulting in insulating behavior. Due to the electron-phonon interaction, phonon mode becomes soft (soft mode) and lattice distortion thus takes place ${ }^{64}$. CDW can also occur in two-dimensional or three-dimensional materials with a nested Fermi surfaces (parallel pieces of Fermi surfaces), which hides 1D character. In this case, the system is no longer truly periodic, since it possesses two or more unrelated periods. And such system is more stable than unmodulated regular lattices. However, there are still subjects of debate, such as the exact mechanism of CDW instability in $\mathrm{NbSe}_{2}{ }^{65}$ and the origin of phase transition in $1 \mathrm{~T}-\mathrm{TaS}_{2}$ and $1 \mathrm{~T}-$ $\mathrm{TaSe}_{2}{ }^{66}$. The discovery of CDW phases in the group $\mathrm{V}$ layered dichalcogenides ${ }^{67,68}$ and new routes to synthesize related lowdimensional TMDs materials lead to renewed interest in this research field ${ }^{5,67}$. Furthermore, CDW phase transition often induces weak variation in the transport properties but exhibits strong optical signatures, especially Raman features, which provides effective method for characterizing phase transition. 

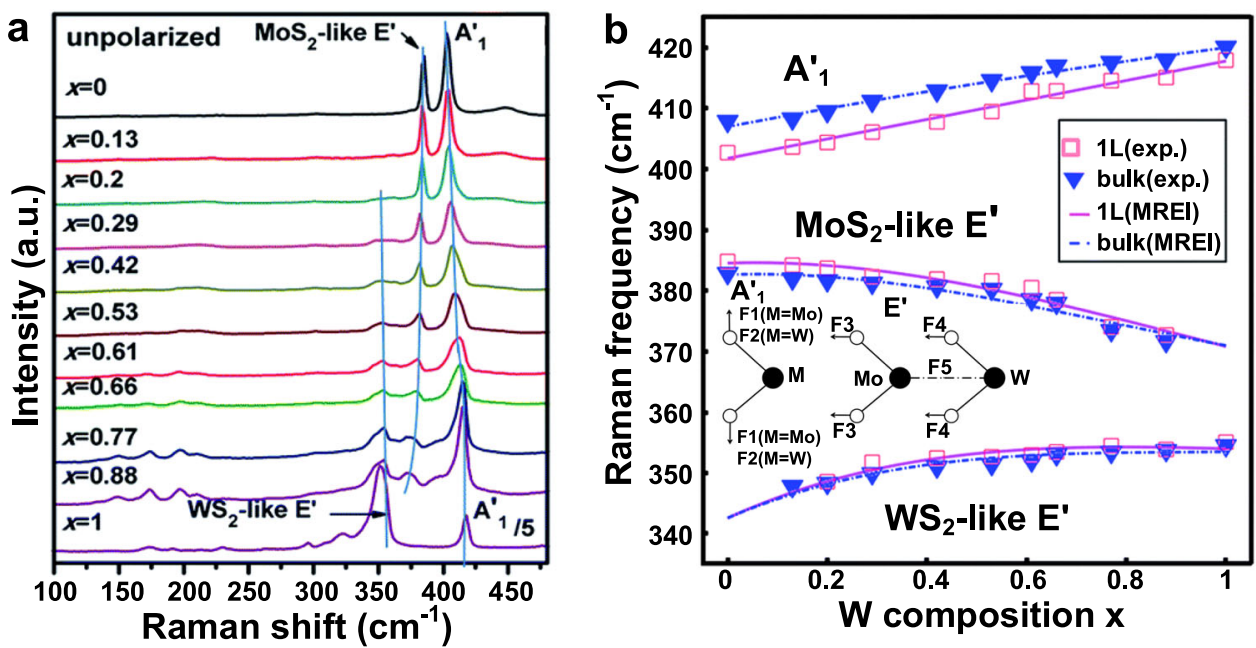

Fig. 4 Alloy effect on Raman spectra. a Raman spectra of $\mathrm{Mo}_{1-x} \mathrm{~W}_{\mathrm{x}} \mathrm{S}_{2}$ monolalyer with different $\mathrm{W}$ composition $x$. The three solid (blue) lines guided by eyes show frequency shift of $E^{\prime}$ and $A_{1}^{\prime}$ modes in $\mathrm{Mo}_{1-x} \mathrm{~W}_{\mathrm{x}} \mathrm{S}_{2}$ monolayer. $\mathbf{b}$ Composition dependent Raman frequency of $E^{\prime}$ and $A_{1}^{\prime}$ ( $E_{2 g}^{1}$ and $A_{1 \mathrm{~g}}$ for bulk) modes in $\mathrm{Mo}_{1-x} \mathrm{~W}_{\mathrm{x}} \mathrm{S}_{2}$ alloys. The solid and dashed lines are the MREl fits of $\mathrm{Mo}_{1-x} \mathrm{~W}_{x} \mathrm{~S}_{2}$ monolayer and bulks, respectively, and the square and triangle indicates experimental data. The insert is the schematics of force constants used in MREl model. Republished with permission of Chen et al. ${ }^{57}$ permission conveyed through Copyright Clearance Center, Inc.

According to relationship between CDW wavelength and lattice constant, CDW can be commensurate, nearly commensurate and incommensurate, which exhibits distinct Raman features. The commensurate phase with distorted structure leads to reconstruction of the lattice, and further results in phonon folding effects, in which the phonons inside the original Brillouin zone (BZ) can fold back to the $\Gamma$ point and be activated in the Raman spectroscopy. However, in incommensurate CDW phase, the loss of translational symmetry and corresponding relaxation of the phonon momentum conservation ${ }^{20,21}$ are similar to that in amorphous materials with broad Raman peaks. This can be as an obvious signature of the CDW phase transition. Accordingly, phase transition between commensurate, nearly commensurate and incommensurate CDW can be probed by Raman spectroscopy. For example, in both 1T$\mathrm{TaS}_{2}{ }^{69}$, and $2 \mathrm{~T}_{-} \mathrm{TaSe}_{2}{ }^{70}$, the sample undergoes a transition from commensurate to incommensurate CDW phase with increasing temperature, and folded back acoustic and optical Raman modes merge and become very broad.

The soft mode and collective excitation of the CDW fluctuations (amplitude mode) in Raman spectra also provide a direct evidence for CDW phase transition ${ }^{71}$. In Fig. 5a, Raman spectra of $\mathrm{NbSe}_{2}$ exhibits a broad feature abound $180 \mathrm{~cm}^{-1}$ at room temperature, which involves a second-order scattering process and is assigned as soft mode. The soft mode redshifts with decreasing temperature and freezes for $T<T_{\mathrm{CDW}}$, where $T_{\mathrm{CDW}}$ indicates CDW transition temperature. Below $T_{\mathrm{CDW}}$, amplitude mode appears, whose intensity $\left(I_{A}\right)$ drops rapidly to zero as temperature approaching $T_{\text {CDW }}$ from below. The frequency of amplitude mode increases monotonically from $\sim 35 \mathrm{~cm}^{-1}$ in bulk to $\sim 70 \mathrm{~cm}^{-1}$ in monolayers. And the $T_{\mathrm{CDW}}$ obtained from $I_{\mathrm{A}}$ as a function of temperature (Fig. $5 c)$ also increases from $36 \pm 1 \mathrm{~K}$ in bulk to $145 \pm 3 \mathrm{~K}$ in monolayers, indicating enhanced electron-phonon interaction in $2 \mathrm{D} \mathrm{NbSe}_{2}$. The results demonstrate that Raman spectroscopy is of great benefit to further explore precise origin of the CDW instability and phase transition.

\section{Magnetism in 2DMs}

At zero temperature, ferromagnetic order in 3D systems can extend over macroscopic length scales, driven by the interaction between the neighboring spins (exchanging coupling) that tends to favor specific relative orientations between them. With temperature above a finite $T_{\mathrm{c}}$, thermal fluctuations tend to destroy long-range order, where $T_{c}$ depends on the effectiveness of thermal fluctuations. For the $2 \mathrm{D}$ case, the situation is more complex. The spin can be constrained along a given direction $(n=$ $1)$, in a given plane $(n=2)$ and completely unconstrained $(n=3)$, and described by 2D Ising, $X Y$ and Heisenberg models, respectively ${ }^{59}$. The atomically magnetic thin crystals are recently found, such as Ising ferromagnet $\mathrm{Crl}_{3}{ }_{3}{ }^{61}$, Heisenberg ferromagnet $\mathrm{Cr}_{2} \mathrm{Ge}_{2} \mathrm{Te}_{6}{ }^{62}$, Ising antiferromagnet $\mathrm{FePS}_{3}{ }^{72,73}$, XY-type antiferromagnet $\mathrm{NiPS}_{3}{ }^{38,74}$. And ferromagnetic order can exist even at room temperature in some ferromagnets, such as $\mathrm{MnSe}_{2}{ }^{75}$ and $\mathrm{VSe}_{2}{ }^{76}$. For the cases of more complex magnetic interactions, more sophisticated models are necessary, such as Kitaev model for frustrated magnetic interactions ${ }^{77}$. All aspects of progress in 2D magnet provide ideal platforms to study the ground state, fundamental excitations, dynamics, and frustrations of spin ensembles under strong quantum confinement.

The magnetic excitation or spin-phonon coupling makes it accessible to measure the magnetic fluctuations and magnons by Raman spectroscopy, such as broad background in $\mathrm{Cr}_{2} \mathrm{Ge}_{2} \mathrm{Te}_{6}$ induced by thermal magnetic fluctuations (above Curie temperature $)^{78}$, spin waves excitation in $\mathrm{Crl}_{3}{ }^{37}$, two-magnon scattering and Fano resonance between phonon and magnon in $\mathrm{NiPS}_{3}{ }^{38}$. Raman spectroscopy can also provide an evidence for long-range magnetic order in 2D magnets. For antiferromagnet magnetic ordering, the doubled unit cell results in BZ folding and induces a series of new Raman modes, which has been used to investigate the Ising-type antiferromagnet $\mathrm{FePS}_{3}{ }^{72,73}$.

In contrast to ordered magnet, Kitaev magnet, with more complex magnetic interactions and strong spin-orbit coupling, are expected to result in a spin-liquid ground state, where quantum fluctuation obstructs long-range order at low temperature $^{77}$. The quantum spin liquid states is one of the most elusive and intriguing topological states, which can be identified in scattering experiments ${ }^{77}$. $a-\mathrm{RuCl}_{3}$ is a prime candidate for fractionalized Kitaev physics, whose unconventional excitations can be observed by polarized Raman scattering ${ }^{79-81}$. The temperature-dependent Raman spectra of $a-\mathrm{RuCl}_{3}$ are presented in Fig. 6a. A broad continuum is observed in the obtained spectrum, as shown in detail in Fig. 6b. Due to the nonmonotonic temperature dependence of integrated intensity $\left(I_{\text {mid }}\right)$ in range of $40-120 \mathrm{~cm}^{-1}$ (Fig. 6c), the continuum is not easily explained by conventional excitation of magnon, electron or phonon, which exhibits monotonic dependence on temperature. Such behavior is 

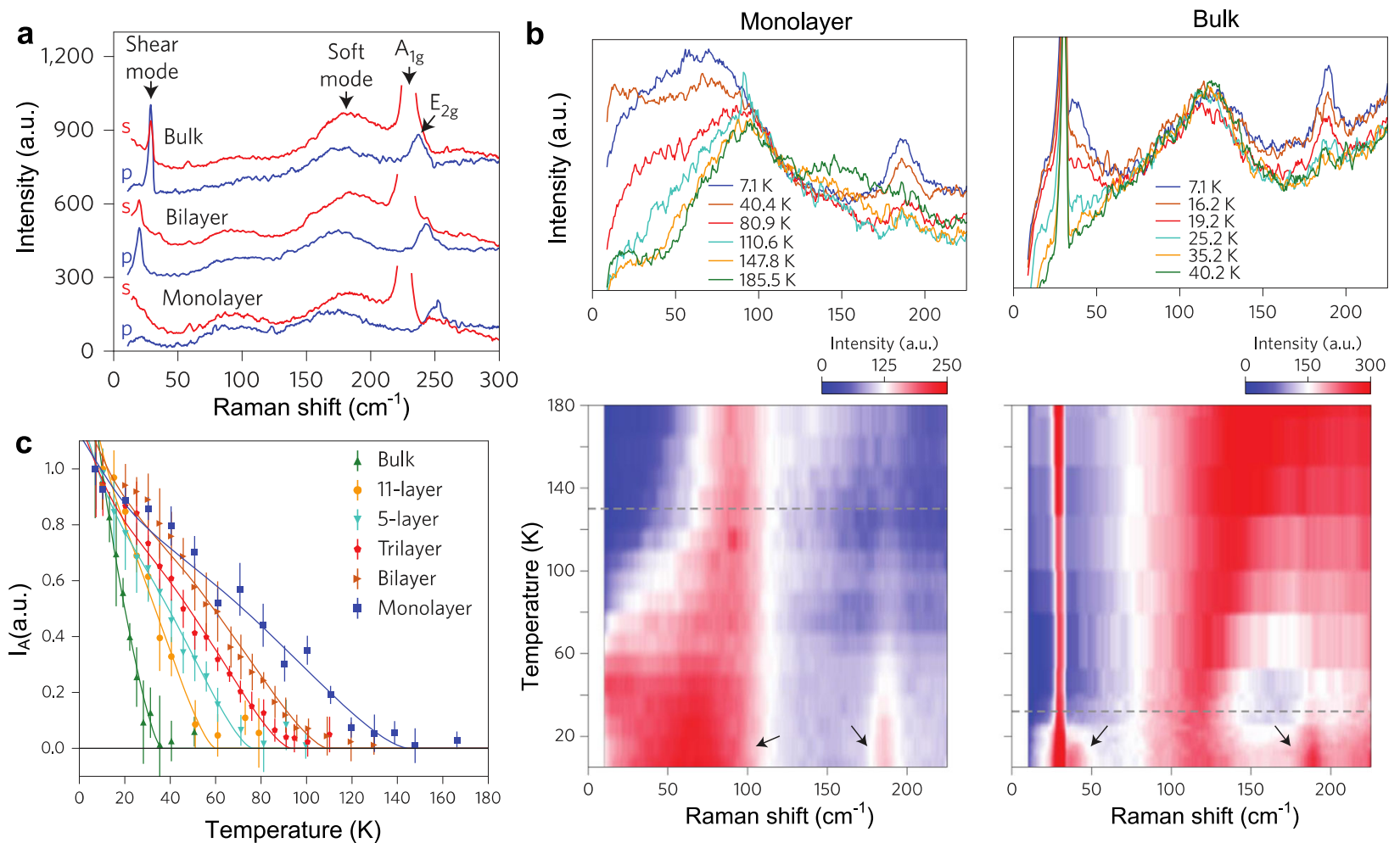

Fig. 5 Thickness-dependent CDW phase transition. a Raman spectra of monolayer, bilayer, and bulk NbSe ${ }_{2}$ at room temperatures for parallel ( $s$, red lines) and perpendicular ( $p$, blue lines) polarization configurations. b Raman spectra of monolayer and bulk NbSe ${ }_{2}$ at selected temperatures and corresponding temperature maps of Raman spectra. The two arrows in each map indicate the amplitude mode (the lowfrequency feature) and the weak high-frequency broad feature. Dashed lines approximately delineate $\mathrm{T}_{\mathrm{CDW}}$. c Temperature-dependent intensity $\left(I_{\mathrm{A}}\right)$ of amplitude mode for $\mathrm{NbSe}_{2}$ samples with different thickness. Images used with permission from Springer Nature ${ }^{69}$.
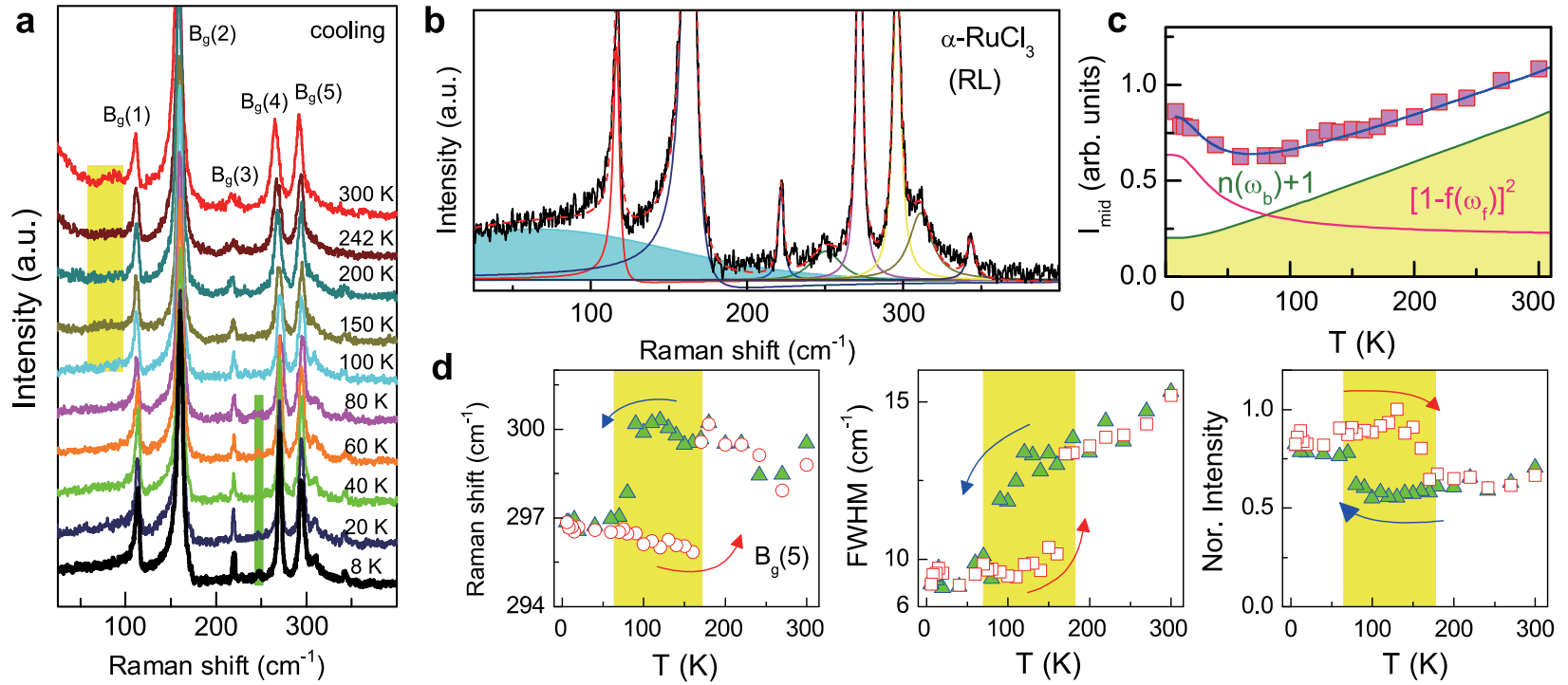

Fig. 6 Magnetic and structural phase transition in $\mathbf{R u C l}_{3}$. a Temperature dependent Raman spectra measured on cooling situation in the inplane (RL) polarization, where incident and scattering light are right and left circular polarization, respectively. The yellow (green) bars marks the modes which disappears (appears) below transition temperature. $\mathbf{b}$ Raman spectra of $a-\mathrm{RuCl}_{3}$ obtained at $T=8 \mathrm{~K}$ fitted by one Gaussian profile (cyan shaded region), two Fano peaks (red and blue solid lines), and six Lorentzian peaks (colored solid lines). c Temperature dependence of $I_{\text {mid. }}$. The shaded area indicates the bosonic background and the solid red line is a fit to temperature dependence of a twofermion creation or annihilation process. $\mathbf{d}$ Thermal hysteresis loop of the frequency, the FWHM and the normalized intensity of $\mathrm{B}_{\mathrm{g}}(5)$ mode. The yellow shaded region indicates the mixture of the monolinic and rhombohedral phase. The arrows indicate the direction of the temperature sweep. Reprinted figures with permission from Glamazda et al. ${ }^{99}$. 
consistent with theoretical predictions for the Kitaev spin liquid ${ }^{79}$, where the temperature dependence (Fig. 6c) involves contributions of magnons and pairs of Majorana fermion. Consequently, the broad continuum in Raman spectra is the evidence of unusual magnetic excitation and a proximate quantum spin liquid ground state. And the Fano line shape of the Raman peaks at 116.6 and $163.7 \mathrm{~cm}^{-1}$ is a signature of spin-phonon coupling. Furthermore, temperature-dependent intensity and FWHM of phonon modes (such as $B_{g}(5)$ in Fig. 6d) with large thermal hysteresis loop indicate first-order structural phase transition from monoclinic to a rhombohedral structure as temperature decreases. And the similar relationship between magnetism and structure phase transition is also observed in $\mathrm{MoCl}_{3}{ }^{82}$, which provides opportunities for revealing magnetic phase transition and lattice engineering to achieve quantum states phase. The results indicate that Raman spectroscopy is a powerful technique to characterize various magnets.

\section{RAMAN SPECTRA OF VDWHS}

In transitional 3D materials, the crystal lattice match between two different materials is necessary for coupling between them in heterostructure, while vdWHs stacked by two different 2DMs or same constituents with a twisted angle go beyonds this limitation ${ }^{9}$. Without direct chemical bonding, the integration of highly disparate materials by vdW interaction make it accessible to create hybrid structures with totally new physics and unique functionality ${ }^{9,10}$. The interlayer coupling is fundamental and plays pivotal role in revealing novelty of vdWHs. The individual constituents, twist angle $(\theta)$, and stacking order of neighboring layers can effectively modify interlayer coupling in vdWHs. It can be characterized by evolution of Raman spectra in vdWHs, which exhibits influence on both intralayer and interlayer Raman modes. To reveal interlayer coupling in vdWHs, here, we discuss moiré phonons in twisted bilayer $\mathrm{MoS}_{2}$ (t2LM), interlayer modes in $\mathrm{MoS}_{2} /$ graphene vdWHs and cross-dimensional electron-phonon coupling (EPC) in $\mathrm{WS}_{2} / \mathrm{hBN}$ vdWHs.

\section{Moiré phonons in $\mathrm{t} 2 \mathrm{LM}$}

The lattice structure of 2DMs can be modified by stacking two layers, or overlaying different layered materials with nearly the same lattice constant with a certain twist angle $\theta$, which forms van deer Waals homo- or heterostructure and generates moiré patterns ${ }^{83}$. The moiré pattern induces a periodic potential based on the patterned interlayer interactions. By varying $\theta$, modified periodic moiré potential further modulates electron-electron interaction, electron-phonon coupling, and phonon-phonon interaction of its constituents ${ }^{11,84-87}$, with emergence of exotic quantum phenomena, such as superconductivity in twisted bilayer graphene (t2LG) with magic angle ${ }^{60}$, moire exciton ${ }^{88}$, and hybridized excitons ${ }^{89}$. For example, in tMLG, interlayer coupling induce van Hove singularities (vHSs) in the joint density of states of all optically allowed transitions, and the intensity of shear mode can be largely enhanced, when the incident excitation energy is resonant with $\mathrm{vHSs}^{31,90}$. Furthermore moiré pattern would result that off-center phonons of constituents are folded to $\Gamma$ point of the moiré superlattices ${ }^{31,91}$, and generate additional Raman active modes, such as the additional $\mathrm{R}$ and $\mathrm{R}^{\prime}$ modes in $\mathrm{t} 2 \mathrm{LG}$ and moiré phonons in $\mathrm{t} 2 \mathrm{LM}^{30,31}$.

Figure 7a presents moiré pattern and crystallographic superlattice of t2LM with different $\theta$. In t2LM, lattice constant $\left(L_{M}\right)$ and reciprocal lattice vector $(g)$ of moiré superlattice as a function of twisted angle $\theta$ can be be described as follows ${ }^{30}$ :

$$
\begin{aligned}
& L_{M}=\frac{a}{2 \sin (\theta) / 2} \\
& \left|g_{M}\right|=2 b \sin (\theta / 2)
\end{aligned},
$$

where $a$ and $b$ are the in-plane lattice constant and absolute reciprocal lattice vectors of $\mathrm{MoS}_{2}$ unit cell, respectively. However, those in crystallographic superlattice are also dependent on the index of $(m, n)$, i.e., $L_{C}=\frac{a|m-n|}{2 \sin (\theta / 2)}$ and $\left|g_{C}\right|=2 b \sin (\theta / 2)$. In general, lattice constant of crystallographic superlattice is equal (such as $\theta=21.79^{\circ}$ ) to or larger (such as $\theta=10.99^{\circ}$ ) than that of moiré superlattice, as shown in Fig. 7a. Both of crystallographic and moiré superlattice can induce phonon folding. From Fig. 7c, the new Raman modes are observed in t2LMs and their frequencies vary monotonously in the region of $0^{\circ}<\theta<30^{\circ}$, which exhibits little dependence on index of $(m, n)$. Consequently, $\theta$ determined momentum of folded phonons is consistent with $\left|g_{M}\right|$, indicating moiré potential induced phonon folding effect. The moiré phonons exhibit significant dependence on twist angle (Fig. 7c) and its frequencies as a function of $\theta$ provide phonon dispersion of $1 \mathrm{~L}$ constituents along the trajectory of $\mathrm{g}$, which exhibits a

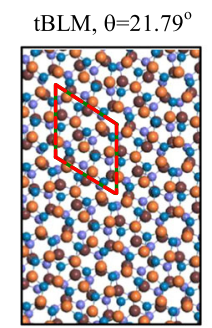

atom S Mo

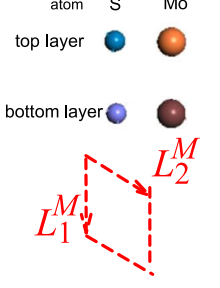

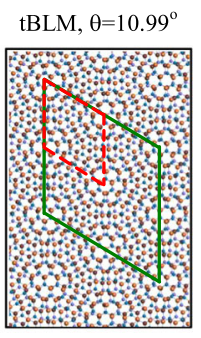

b

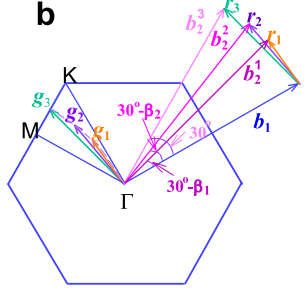

C

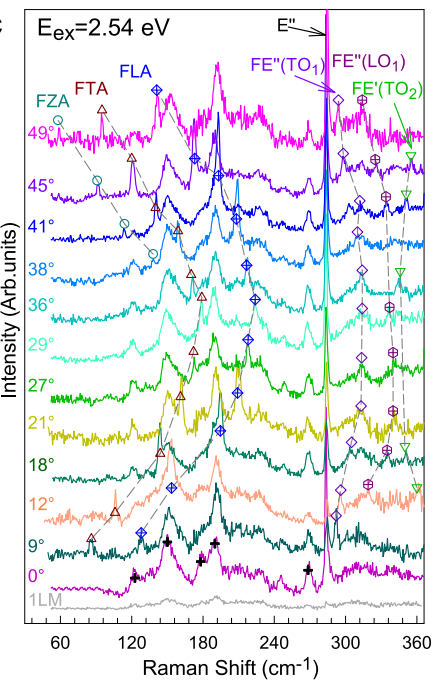

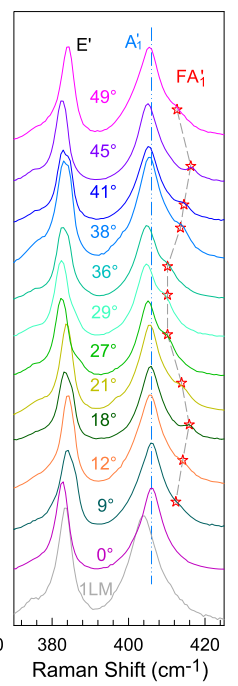

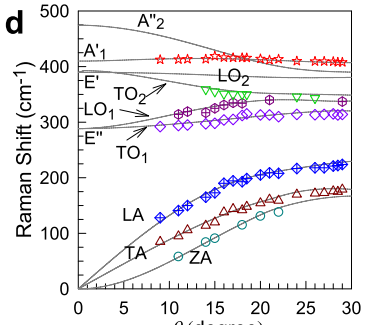

e $500 \frac{\theta(d e g}{\mathrm{A}^{\prime \prime 2}}$

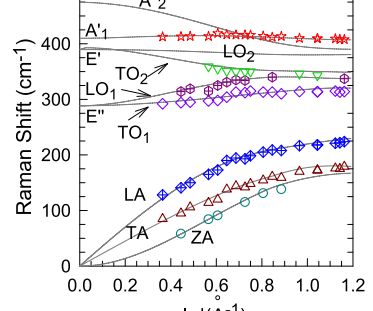

$|\mathbf{g}|\left(\mathrm{A}^{-1}\right)$

Fig. 7 Moiré phonons. a Crystallographic (green solid lines) and moiré superlattices (red dashed lines) in t2LM with $\theta=21.79^{\circ}$ and $t 2 \mathrm{LM}$ with $\theta=10.99^{\circ}$. b Schematic diagram of moire basic vectors $\left(g_{\mathrm{i}}, i=1,2,3\right)$ when $\theta \leq 30^{\circ}$. c The evolution of Raman spectra as a function of $\theta$ in the region of $50-365$ and $370-425 \mathrm{~cm}^{-1}$. The Raman modes belonging to different phonon branches are presented by symbols with different shapes and color. The Raman spectra of $1 \mathrm{~L}$ and $3 \mathrm{R}$-bilayer $\left(\theta=0^{\circ}\right) \mathrm{MoS}_{2}$ are also presented. The frequency comparison between theoretically calculated and experimental frequency of moiré phonons dependent on $\theta(\mathbf{d})$, and $|g|(\mathbf{e})$. Reprinted with permission from Lin et al. ${ }^{30}$. 
excellent agreement with theory calculation (Fig. 7d, e) and confirm moiré pattern induced phonon folding effect. The t2LMs with different angle provide possibility to access entire phonon branches by Raman spectra, which also provides potential application in other 2DMs and vdWHs. We note that the superlattice generated by ion intercalation can also induce phonon folding and additional resonance effect, such as lithiumintercalated $\mathrm{NiPS}_{3}{ }^{92}$ and $\mathrm{MS}_{3}(\mathrm{M}=\mathrm{Ti}, \mathrm{Zr}, \mathrm{Hf})^{93}$.

\section{Interlayer coupling in vdWHs}

For the vdWHs formed by two 2DMs with distinct properties, the interfacical coupling in vdWHs is important for fundamental properties and application of entire vdWHs. Figure $8 a-c$ present the preparation process of vdWHs and its schematic of crystal structure, formed by bilayer $\mathrm{MoS2}_{2}$ (2LM) and $1 \mathrm{LG}$, denoted as $2 \mathrm{LM} / 1 \mathrm{LG}$. Figure $8 \mathrm{~d}$ presents frequency mapping images of $\mathrm{LB}_{2,1}$ peaks of $2 \mathrm{LM} / 1 \mathrm{LG}$ vdWHs, where interfacial coupling exhibits significant influence on frequency of LB mode. From the Raman spectra of $2 \mathrm{LM} / n \mathrm{LG}$ vdWHs in Fig. 8, three branches of LB mode $\left(\mathrm{LB}_{\mathrm{N}, \mathrm{N}-1}, \mathrm{LB}_{\mathrm{N}, \mathrm{N}-2}\right.$, and $\left.\mathrm{LB}_{\mathrm{N}, \mathrm{N}-3}\right)$ are observed and exhibits obvious red-shift as increasing thickness of graphene, where $\mathrm{N}$ indicate total layer number of $\mathrm{MoS}_{2}$ and graphene. In general, only nearest LB force constant of MoS2 $\left(a^{\perp}(M)=84 \times 10^{18} \mathrm{~N} \mathrm{~m}^{-3}\right)^{94}$, nearest $\left(\alpha^{\perp}(G)=106 \times 10^{18} \mathrm{~N} \mathrm{~m}^{-3}\right)$ and second nearest $\left(\beta^{\perp}(G)=9.3 \times 10^{18}\right.$ $\mathrm{N} \mathrm{m}^{-3}$ ) LB force constant of graphene ${ }^{32}$ are respectively involved in LCM of multilayer $\mathrm{MoS}_{2}$ and graphene. However in vdWHs, graphene and $\mathrm{MoS}_{2}$ should be considered as an overall system, and the nearest interfacical LB coupling between $\mathrm{MoS}_{2}$ and grpahene $\left(a^{\perp}(I)=60 \times 10^{18} \mathrm{~N} \mathrm{~m}^{-3}\right)$ is necessary, as shown in insert of Fig. 8. The modified LCM can well reproduce all experimental frequency of LB mode as a function of thicknessf of $\mathrm{MOS}_{2}$ and graphene, as shown in Fig. 8f, g. Furthermore, the interfacial layerbreathing coupling is found to be insensitive to stacking order and twist angle. Therefore, the interfacial interactions in vdWHs can be well estimated by Raman spectroscopy.

As shown in Fig. 8d, S mode of $2 \mathrm{LM} / \mathrm{nLG}$ shows almost same frequency to that of $2 \mathrm{LM}$ and exhibits little dependence on layer number of graphene, indicating weak interfacial shear coupling. In other vdWHs, such as tMLG, similar results are also found. Consequently, interfacial LB coupling in vdWHs can induce new LB modes, and can be employed for interfacial interaction and identify the total thickness of vdWHs, while shear mode is localized in the individual constituents and can be used for their thickness.

\section{Cross-dimensional EPC in vdWHs}

In vdWHs, interlayer coupling would result in interaction between elementary excitations in 2DMs and vdWHs, where many-body effect significantly renoramlizes related properties and leads to new quantum phenomena. Especially, the electron-phonon coupling plays a key role in quantum phenomena in condensed matter physics and generate numerous fascinating physical effects, such as excitation of electron and phonon coupled states in multilayer graphene ${ }^{95}$. Especially for vdWHs, involved constituents and twist angle $\theta$ provide extra freedom for manipulating many-body effect. Recently, the intralayer and interlayer EPC are observed in $\mathrm{t} 2 \mathrm{LG}^{84}$ and $\mathrm{WSe}_{2} /$ hBN vdWHs ${ }^{27}$, which involves electrons in the same and adjacent layers, respectively. The interpreted mechanism of interlayer EPC would provides further control of the physical properties of vdWHs.
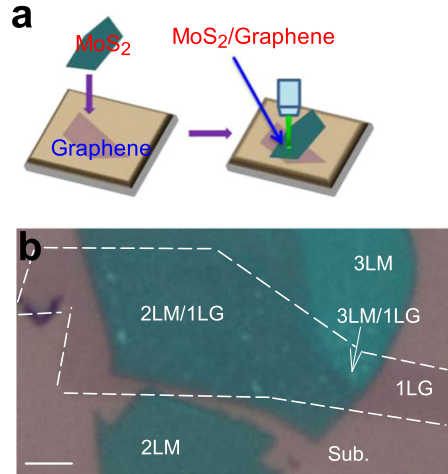

C
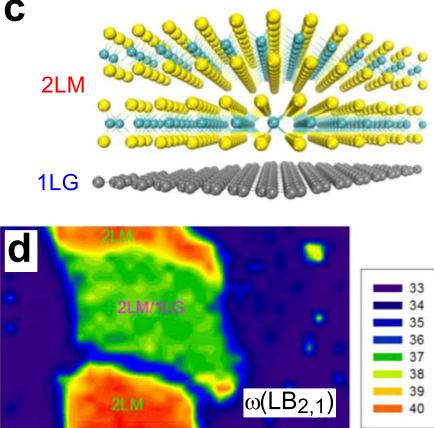

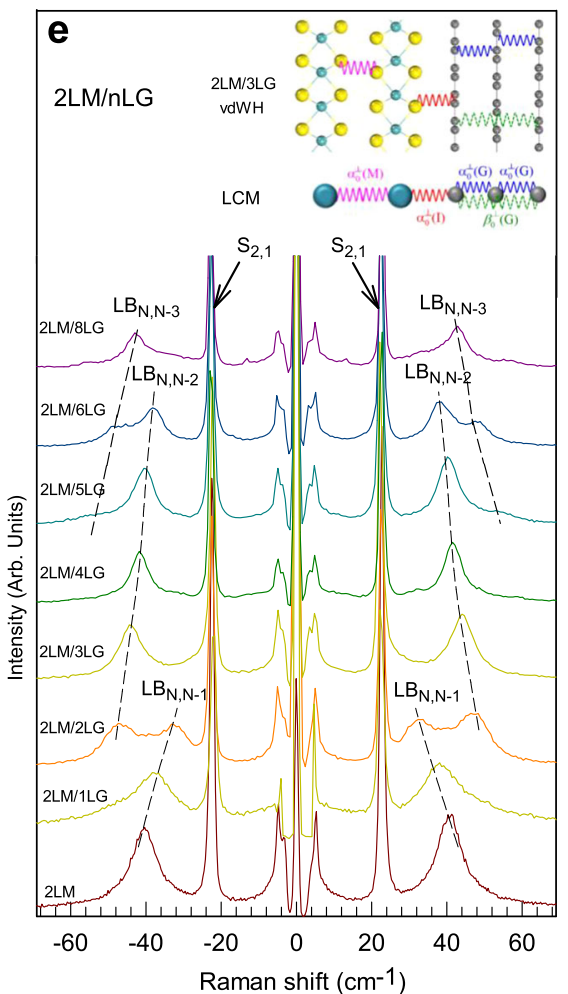

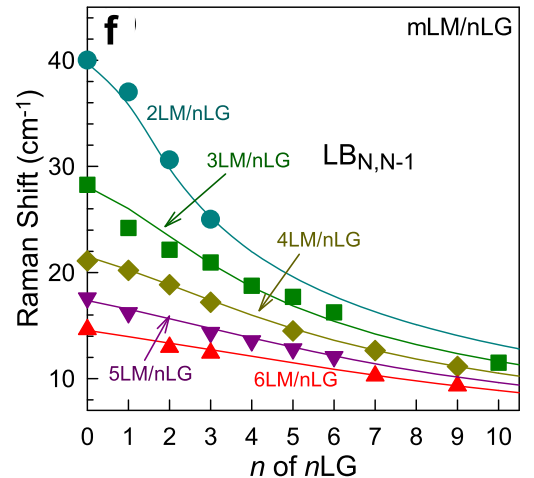

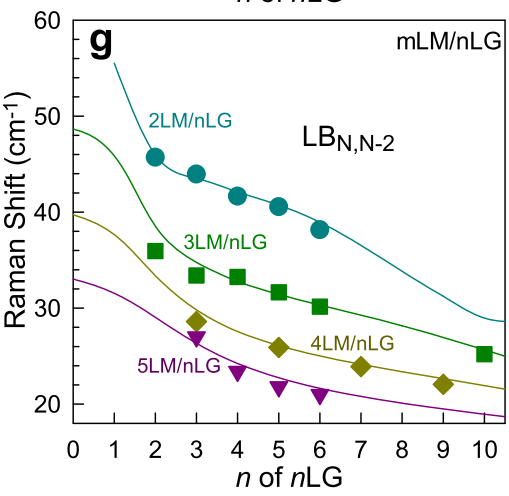

Fig. 8 Interlayer Raman modes in graphene/MoS $\mathbf{2}$ vdWHs. a Schematic of preparation of $\mathrm{MoS}_{2} /$ graphene vdWHs. b Optical image of the vdWHs formed by $2 \mathrm{LM}$ and $3 \mathrm{LM}$ on $1 \mathrm{LG}$. Scale bar, $10 \mu \mathrm{m}$. The white dash line indicates $1 \mathrm{LG}$ zone. c Crystal structure of vdWHs formed by $2 \mathrm{LM}$ and 1LG. d Frequency maps of LB mode. e Raman spectra of pristine 1LG, as-transferred $1 \mathrm{LG}$ and $2 \mathrm{LM} / 1 \mathrm{LG}$, and 2LM/1LG vdWHs annealed for 5,30 , and $60 \mathrm{~min}$. The insert illustrates structure of $2 \mathrm{LM} / 1 \mathrm{LG}$ vdWHs. $\mathrm{f}$ Raman of $2 \mathrm{LM} / \mathrm{nLG}(n=0-8)$ in the shear and LB peak region. The insert presents LCM for LB modes in $2 \mathrm{LM} / 3 \mathrm{LG}$, considered with the nearest $L B$ coupling in the $3 \mathrm{LG}$ constituents. Pos $\left(\mathrm{LB}_{\mathrm{N}, \mathrm{N}-1}\right) \mathbf{g}$ and Pos $\left(\mathrm{LB}_{\mathrm{N}, \mathrm{N}-2}\right) \mathbf{h}$ as a function of n-layer graphene, fitted by linear chain model(solid lines). Reprinted with permission from Li et al. ${ }^{34}$. 

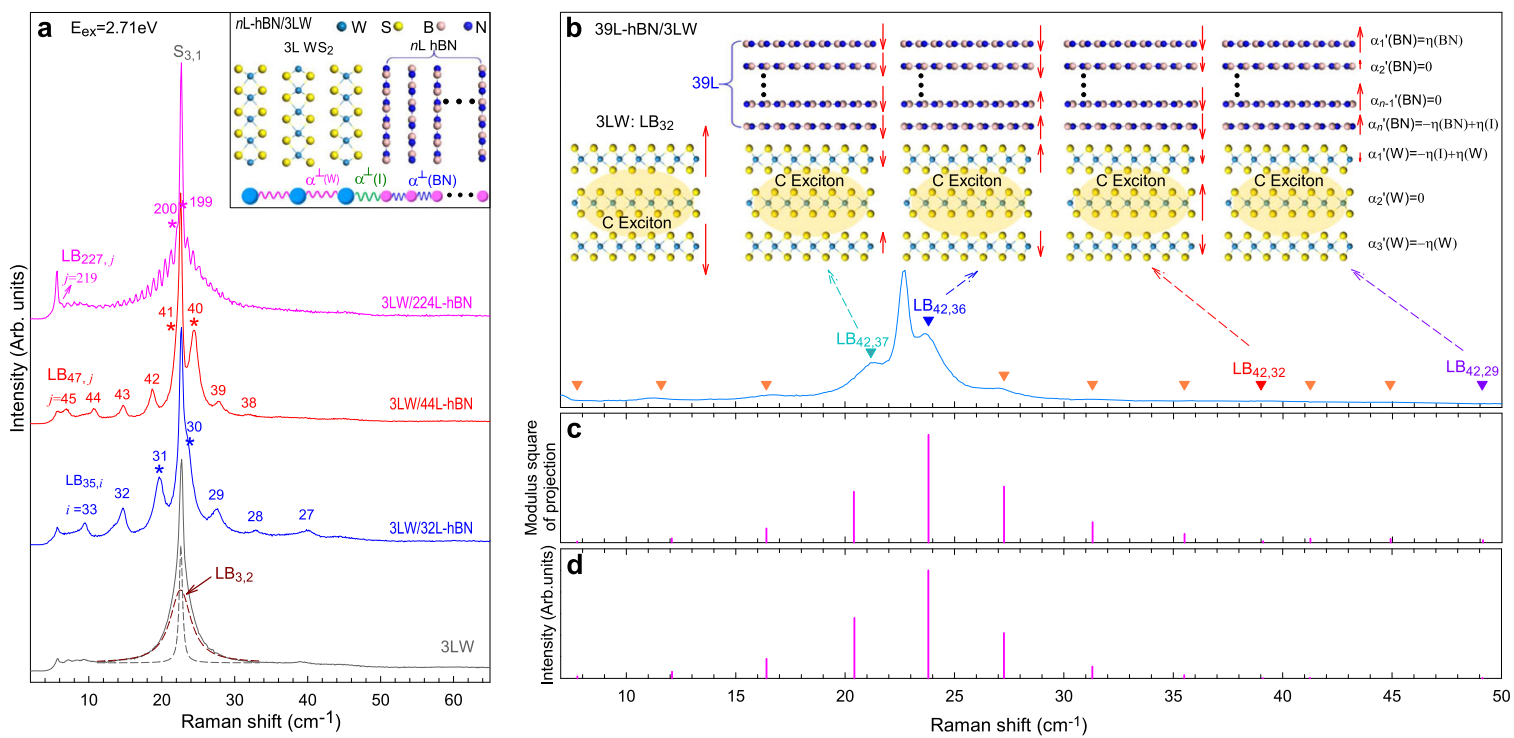

Fig. 9 Interlayer electron-phonon coupling of LB modes in $\mathbf{W S}_{\mathbf{2}} / \mathbf{h B N}$ vdWHs. a Raman spectra of 3LW/32L-hBN, 3LW/44L-hBN, and 3LW/ 224L-hBN along with the standalone 3LW flake. The spectra is scaled and offset for clarify. The stars represent the two prime LB modes in vdWHs. b Raman spectra of a $39 \mathrm{~L}-\mathrm{hBN} / 3 \mathrm{LW}$ in the region of $5-50 \mathrm{~cm}^{-1}$ and the normal mode displacements (red arrows) of the $\mathrm{LB}_{42,37}, \mathrm{LB}_{42,36}$, $\mathrm{LB}_{42,32}$, and $\mathrm{LB}_{42,29}$ modes in a $39 \mathrm{~L}-\mathrm{hBN} / 3 \mathrm{LW}$ and the $\mathrm{LB}_{3,2}$ in a standalone $3 \mathrm{LW}$ flake. The triangles represent the representative expected $\mathrm{LB}$ modes in the 39L-hBN/3LW based on the LCM, shown in the inset. $\mathbf{c}$ The modulus square of the projection from wavefunction of different LB modes in 39L-hBN/3LW vdWH onto the wavefunction of the $\mathrm{LB}_{3,2}$ mode in a standalone $3 \mathrm{LW}$ flake. $\mathbf{d}$ The relative intensity of $\mathrm{LB}$ modes in $39 \mathrm{~L}-$ $\mathrm{hBN} / 3 \mathrm{LW}$ vdWH based on the interlayer bond polarizability model. Images used with permission form Springer Nature ${ }^{35}$.

Figure 9a presents a comparison of standalone $3 \mathrm{~L} \mathrm{WS}_{2}$ (3LW) and vdWHs formed by $3 \mathrm{LW}$ and $\mathrm{nL} h \mathrm{hBN}(\mathrm{nL}-\mathrm{hBN}, n=32,44$, and 224), where additional Raman modes from the collective LB vibrations of all the stacking layers in vdWHs are observed ${ }^{35}$. According to modified LCM in vdWHs shown in Fig. 9a, LB modes in $\mathrm{WS}_{2} / \mathrm{hBN}$ vdWHs can be reproduced well by involving the interlayer LB coupling of $\mathrm{WS}_{2}\left(a^{\perp}(W)=90 \times 10^{18} \mathrm{~N} \mathrm{~m}^{-3}\right)^{96}$, the interlayer LB coupling of $\mathrm{hBN}\left(\alpha^{\perp}(\mathrm{BN})=98.8 \times 10^{18} \mathrm{~N} \mathrm{~m}^{-3}\right)$ and the interfacial coupling between $n \mathrm{~L}-\mathrm{hBN}$ and $\mathrm{mLW}\left(a^{\perp}(I)=\right.$ $89.7 \times 10^{18} \mathrm{~N} \mathrm{~m}^{-3}$ ). The observed LB mode of vdWHs over tens to hundreds of layer thickness should exhibit bulk-like feature, which is attributed to the coupling between layer-localized twodimensional electrons confined to the few-layer $\mathrm{WS}_{2}$ constituents and bulk-like LB phonons extended over the entire vdWHs, socalled cross-dimensional EPC. Furthermore, LB modes are resonantly enhanced as excitation energy close to the $C$ exciton energy of standalone MLW flakes, suggesting these 3D LB modes can strongly couple to the two-dimensional electron states confined within the multilayer $\mathrm{WS}_{2}(\mathrm{mLW})$ constituents.

To put insight into the cross-dimensional EPC, a microscopic picture mediated by the interfacial coupling and the interlayer bond polarizability model in vdWHs are proposed ${ }^{35}$. According to a microscopic picture mediated by the interfacial coupling, the $L B$ vibrations in $\mathrm{mLW}$ constituent can efficiently interact with those in $\mathrm{nL}-\mathrm{hBN}$ constituent, leading to the bulk-like LB vibrations. The relative Raman intensity can be obtained by the square of projection between the vdWH LB phonon wavefunction $(\Psi)$ and that $\left(\varphi_{\mathrm{j}}\right)$ of $\mathrm{LB}_{\mathrm{m}, \mathrm{m}-\mathrm{j}}(j=1,2, \ldots m-1)$ phonon in a standalone $\mathrm{mLW}$, i.e., $p^{2}=\sum_{\mathrm{j}} \rho_{\mathrm{j}} p_{\mathrm{j}}^{2}$, where $p_{\mathrm{j}}=$ $\left|\left\langle\varphi_{\mathrm{j}} \mid \psi\right\rangle\right|$ and $\rho_{\mathrm{j}}$ is the relative Raman intensity of the corresponding $L B_{m, m-j}$ in the standalone $m L W$, in excellent agreement with experimental results as shown in Fig. 9c. Additionally, the interlayer bond polarizability model can also achieve relative intensity by the square of system polarizability change $\left(a^{2}\right)$, which is related to interlayer bond polarizability and bond

$$
\begin{aligned}
& \text { vector }^{97} \\
& \qquad \begin{aligned}
\Delta a= & \sum_{i} a_{i} \Delta z_{\mathrm{i}} \\
= & \eta(\mathrm{W})\left[\Delta z_{1}(\mathrm{~W})-\Delta z_{3}(\mathrm{~W})\right] \\
& +\eta(\mathrm{BN})\left[\Delta z_{1}(\mathrm{BN})-\Delta z_{n}(\mathrm{BN})\right] \\
& +\eta(\mathrm{I})\left[\Delta z_{n}(\mathrm{BN})-\Delta z_{1}(\mathrm{~W})\right]
\end{aligned}
\end{aligned}
$$

where $a_{\mathrm{i}}$ is the polarizablity derivative of the entire layer $i$ with respective to the direction normal to the basal plane $(z)$ and $\Delta z_{i}$ is the normal displacement of layer $i$ from the LCM, $\eta(\mathrm{W}), \eta(\mathrm{BN})$, and $\eta(\mathrm{I})$ are fitting parameters related to the properties of the interlayer bond in $\mathrm{WS}_{2}, \mathrm{hBN}$ and the interface. The Raman intensities of LB modes in vdWHs can be well reproduced with $\eta$ $(\mathrm{I}) / \eta(\mathrm{W})=0.3$ and $\eta(\mathrm{BN}) / \eta(\mathrm{I})=0.003$, as shown in Fig. $9 \mathrm{~d}$. The results reveal cross-dimensional EPC in vdWHs, and demonstrate a new access to manipulate both the designable phonon excitations in vdWHs and their coupling to the electronic states by varying the constituents and engineering the interface.

\section{OUTLOOK}

This review presents application of Raman spectroscopy which has been widely used to determine thickness, stacking order, twist angle in vdWHs, interlayer coupling, disorder and strain in 2DMs and vdWHs. And the response to external perturbations, such as temperature, strain, electron doping, provide possible routines to access phase transition, elastic properties, CDW states. With further exploring 2DMs, magnetic properties are observed in 2DMs, where mono-layer or few-layer 2DMs exhibit significant difference compared to their bulk counterparts. The Raman spectroscopy can be as a convenient and non-damage tool to characterize them. 
The 2DMs with increasing type provide large number of possible heterostructure involving combination of them, which make it possible to modify properties of 2DMs and even combine advantage of different 2DMs to designable quantum phenomena. For example, heterostructure based on ferromagnetic materials such as $\mathrm{WSe}_{2} / \mathrm{Crl}_{3}$ strongly affect the valley polarization of $\mathrm{WSe}_{2}{ }^{98}$. The influence of interlayer coupling, electron transfer, spin-phonon coupling would significantly modify properties and is important to understand mechanism of interaction in vdWHs. Raman modes involving the electron, phonon, or magneton scattering and coupling between them could provide information about electron band structure, interlayer coupling in aligned vdWHs. Utilizing Raman spectroscopy, further investigation for unexplored quantum phenomena in 2DMs and modified properties in vdWHs will continue to be spotlighted in condensed matter physics.

\section{DATA AVAILABILITY}

The data that support the findings of this study are available from the corresponding author upon reasonable request.

Received: 7 December 2019; Accepted: 10 March 2020; Published online: 26 May 2020

\section{REFERENCES}

1. Collongues, R. et al. $\beta$ alumina, a typical solid electrolyte: latest developments in fundamental approach and in battery utilization. J. Phys. Chem. Solids 45, 981-1013 (1984).

2. Goodenough, J. B. Rechargeable batteries: challenges old and new. J Solid State Electrochem. 16, 2019-2029 (2012).

3. Novoselov, K. S. et al. Electric field effect in atomically thin carbon films. Science 306, 666-669 (2004).

4. Geim, A. K. Graphene: status and prospects. Science 324, 1530-1534 (2009).

5. Manzeli, S., Ovchinnikov, D., Pasquier, D., Yazyev, O. V. \& Kis, A. 2D transition metal dichalcogenides. Nat. Rev. Mater. 2, 17033 (2017).

6. Tan, C. et al. Recent advances in ultrathin two-dimensional nanomaterials. Chem Rev. 117, 6225-6331 (2017).

7. Ferrari, A. C. et al. Science and technology roadmap for graphene, related twodimensional crystals, and hybrid systems. Nanoscale 7, 4598-4810 (2015).

8. Tan, P.-H. Raman Spectroscopy of Two-Dimensional Materials (Springer, 2019).

9. Geim, A. K. \& Grigorieva, I. V. Van der waals heterostructures. Nature 499, 419 (2013).

10. Novoselov, K., Mishchenko, A., Carvalho, A. \& Neto, A. C. 2 D materials and van der Waals heterostructures. Science 353, aac9439 (2016).

11. Wu, J.-B., Lin, M.-L., Cong, X., Liu, H.-N. \& Tan, P.-H. Raman spectroscopy of graphene-based materials and its applications in related devices. Chem. Soc. Rev. 47, 1822-1873 (2018).

12. Jorio, A., Dresselhaus, M. S., Saito, R. \& Dresselhaus, G. Raman Spectroscopy in Graphene Related Systems (Wiley, 2011).

13. Tan, P. et al. The shear mode of multilayer graphene. Nat. Mater. 11, 294 (2012).

14. Liu, H.-N., Cong, X., Lin, M.-L. \& Tan, P.-H. The intrinsic temperature-dependent Raman spectra of graphite in the temperature range from $4 \mathrm{~K}$ to $1000 \mathrm{~K}$. Carbon 152, 451-458 (2019).

15. Bonini, N., Lazzeri, M., Marzari, N. \& Mauri, F. Phonon anharmonicities in graphite and graphene. Phys. Rev. Lett. 99, 176802 (2007).

16. Pisana, S. et al. Breakdown of the adiabatic Born-Oppenheimer approximation in graphene. Nat. Mater. 6, 198 (2007).

17. Beams, R., Cançado, L. G. \& Novotny, L. Raman characterization of defects and dopants in graphene. J. Phys. Condens. Matter. 27, 083002 (2015)

18. Lee, J.-U. et al. Strain-shear coupling in bilayer $\mathrm{MoS}_{2}$. Nat. Commun. 8, 1370 (2017).

19. Mohiuddin, T. et al. Uniaxial strain in graphene by Raman spectroscopy: G peak splitting, grüneisen parameters, and sample orientation. Phys. Rev. B 79, 205433 (2009).

20. Lin, T., Cong, X., Lin, M.-L., Liu, X.-L. \& Tan, P.-H. The phonon confinement effect in two-dimensional nanocrystals of black phosphorus with anisotropic phonon dispersions. Nanoscale 10, 8704-8711 (2018)

21. Shi, W. et al. Phonon confinement effect in two-dimensional nanocrystallites of monolayer $\mathrm{MoS}_{2}$ to probe phonon dispersion trends away from Brillouin-zone center. Chin. Phys. Lett. 33, 057801 (2016).

22. Shi, W. et al. Raman and photoluminescence spectra of two-dimensional nanocrystallites of monolayer $\mathrm{WS}_{2}$ and $\mathrm{WSe}_{2} .2 D$ Mater 3, 025016 (2016).
23. Merlen, A., Buijnsters, J. G. \& Pardanaud, C. A guide to and review of the use of multiwavelength Raman spectroscopy for characterizing defective aromatic carbon solids: from graphene to amorphous carbons. Coatings 7, 153 (2017).

24. Ando, T. Magnetic oscillation of optical phonon in graphene. J. Phys. Soc. Jpn 76 024712-024712 (2007).

25. Goerbig, M., Fuchs, J.-N., Kechedzhi, K. \& Falko, V. I. Filling-factor-dependent magnetophonon resonance in graphene. Phys. Rev. Lett. 99, 087402 (2007).

26. Ferrari, A. C. \& Basko, D. M. Raman spectroscopy as a versatile tool for studying the properties of graphene. Nat. Nanotechnol 8, 235 (2013).

27. Jin, C. et al. Interlayer electron-phonon coupling in $\mathrm{WSe}_{2} / \mathrm{hBN}$ heterostructures. Nat. Phys. 13, 127 (2017).

28. Cong, X. et al. Probing the acoustic phonon dispersion and sound velocity of graphene by Raman spectroscopy. Carbon 149, 19-24 (2019).

29. Cong, X. et al. Stokes and anti-Stokes Raman scattering in mono-and bilayer graphene. Nanoscale 10, 16138-16144 (2018).

30. Lin, M.-L. et al. Moire phonons in twisted bilayer $\mathrm{MoS}_{2}$. ACS Nano. 12, 8770-8780 (2018).

31. Wu, J.-B. et al. Resonant Raman spectroscopy of twisted multilayer graphene. Nat. Commun. 5, 5309 (2014)

32. $\mathrm{Wu}$, J.-B. et al. Interface coupling in twisted multilayer graphene by resonant Raman spectroscopy of layer breathing modes. ACS Nano. 9, 7440-7449 (2015).

33. Liang, L. et al. Low-frequency shear and layer-breathing modes in Raman scattering of two-dimensional materials. ACS Nano. 11, 11777-11802 (2017).

34. $\mathrm{Li}, \mathrm{H}$. et al. Interfacial interactions in van der waals heterostructures of $\mathrm{MoS}_{2}$ and graphene. ACS Nano. 11, 11714-11723 (2017).

35. Lin, M.-L. Cross-dimensional electron-phonon coupling in van der Waals heterostructures. Nat. Commun. 10, 2419 (2019).

36. Devereaux, T. P. \& Hackl, R. Inelastic light scattering from correlated electrons. Rev. Modern Phys. 79, 175 (2007).

37. Jin, W. et al. Raman fingerprint of two terahertz spin wave branches in a twodimensional honeycomb Ising ferromagnet. Nat. Commun. 9, 5122 (2018).

38. Kim, K. et al. Suppression of magnetic ordering in XXZ-type antiferromagnetic monolayer $\mathrm{NiPS}_{3}$. Nat. Commun. 10, 345 (2019).

39. Zhang, X., Tan, Q.-H., Wu, J.-B., Shi, W. \& Tan, P.-H. Review on the Raman spec troscopy of different types of layered materials. Nanoscale 8, 6435-6450 (2016).

40. Yoon, D., Son, Y.-W. \& Cheong, H. Negative thermal expansion coefficient of graphene measured by Raman spectroscopy. Nano Lett. 11, 3227-3231 (2011).

41. Chen, S.-Y., Goldstein, T., Venkataraman, D., Ramasubramaniam, A. \& Yan, J. Activation of new Raman modes by inversion symmetry breaking in type II Weyl semimetal candidate T'-MoTe 2 . Nano Lett. 16, 5852-5860 (2016).

42. Yang, H., Kim, S. W., Chhowalla, M. \& Lee, Y. H. Structural and quantum-state phase transitions in van der Waals layered materials. Nat. Phys. 13, 931 (2017).

43. Voiry, D., Mohite, A. \& Chhowalla, M. Phase engineering of transition metal dichalcogenides. Chem. Soc. Rev. 44, 2702-2712 (2015).

44. Kappera, R. et al. Phase-engineered low-resistance contacts for ultrathin $\mathrm{MoS}_{2}$ transistors. Nat. Mater. 13, 1128 (2014).

45. Wang, Y. et al. Structural phase transition in monolayer $\mathrm{MoTe}_{2}$ driven by electrostatic doping. Nature 550, 487 (2017).

46. Zhu, J. et al. Argon plasma induced phase transition in monolayer $\mathrm{MoS}_{2}$. J. Am. Chem. Soc. 139, 10216-10219 (2017).

47. Ling, X. et al. Low-frequency interlayer breathing modes in few-layer black phosphorus. Nano Lett. 15, 4080-4088 (2015).

48. Zhang, X. et al. Phonon and Raman scattering of two-dimensional transition metal dichalcogenides from monolayer, multilayer to bulk material. Chem. Soc. Rev. 44, 2757-2785 (2015).

49. Song, Q. et al. Physical origin of Davydov splitting and resonant Raman spectroscopy of davydov components in multilayer MoTe. Phys. Rev. B 93, 115409 (2016).

50. Stenger, I. et al. Low frequency raman spectroscopy of few-atomic-layer thick hBN crystals. 2D Mater 4, 031003 (2017).

51. Verble, J. \& Wieting, T. Lattice mode degeneracy in $\mathrm{MoS}_{2}$ and other layer compounds. Phys. Rev. Lett. 25, 362 (1970).

52. Ghosh, P. N. \& Maiti, C. Interlayer force and Davydov splitting in $2 \mathrm{H}-\mathrm{MoS}_{2}$. Phys. Rev. B 28, 2237 (1983).

53. Molina-Sanchez, A. \& Wirtz, L. Phonons in single-layer and few-layer $\mathrm{MoS}_{2}$ and $\mathrm{WS}_{2}$. Phys. Rev. B 84, 155413 (2011).

54. Tan, Q.-H., Zhang, X., Luo, X.-D., Zhang, J. \& Tan, P.-H. Layer-number dependent high-frequency vibration modes in few-layer transition metal dichalcogenides induced by interlayer couplings. J. Semicond. 38, 031006 (2017).

55. Ning, C.-Z., Dou, L. \& Yang, P. Bandgap engineering in semiconductor alloy nanomaterials with widely tunable compositions. Nat. Rev. Mater 2, 17070 (2017).

56. Zhang, M. et al. Two-dimensional molybdenum tungsten diselenide alloys: photoluminescence, Raman scattering, and electrical transport. ACS Nano. 8, 7130-7137 (2014).

57. Chen, $\mathrm{Y}$. et al. Composition-dependent Raman modes of $\mathrm{Mo}_{1-\mathrm{x}} \mathrm{W}_{\mathrm{x}} \mathrm{S}_{2}$ monolayer alloys. Nanoscale 6, 2833-2839 (2014). 
58. Qiao, X.-F. et al. Substrate-free layer-number identification of two-dimensional materials: a case of $\mathrm{Mo}_{0.5} \mathrm{~W}_{0.5} \mathrm{~S}_{2}$ alloy. Appl. Phys. Lett. 106, 223102 (2015).

59. Burch, K. S., Mandrus, D. \& Park, J.-G. Magnetism in two-dimensional van der Waals materials. Nature 563, 47 (2018).

60. Cao, Y. et al. Unconventional superconductivity in magic-angle graphene superlattices. Nature 556, 43 (2018).

61. Huang, B. et al. Layer-dependent ferromagnetism in a van der Waals crystal down to the monolayer limit. Nature 546, 270 (2017).

62. Gong, C. et al. Discovery of intrinsic ferromagnetism in two-dimensional van der Waals crystals. Nature 546, 265 (2017).

63. Peierls, R. E. Quantum Theory of Solids. (Oxford University, New York/London, 1955).

64. Chan, S.-K. \& Heine, V. Spin density wave and soft phonon mode from nesting Fermi surfaces. J. Phys. F 3, 795 (1973).

65. Arguello, C. et al. Visualizing the charge density wave transition in $2 \mathrm{H}_{-} \mathrm{NbSe}_{2}$ in real space. Phys. Rev. B 89, 235115 (2014).

66. Bovet, M. et al. Pseudogapped Fermi surfaces of $1 \mathrm{~T}-\mathrm{TaS}_{2}$ and $1 \mathrm{~T}-\mathrm{TaSe}_{2}$ : A charge density wave effect. Phys. Rev. B 69, 125117 (2004).

67. Wilson, J., Di Salvo, F. \& Mahajan, S. Charge-density waves in metallic, layered, transition-metal dichalcogenides. Phys. Rev. Lett. 32, 882 (1974).

68. Wilson, J. A., Di Salvo, F. \& Mahajan, S. Charge-density waves and superlattices in the metallic layered transition metal dichalcogenides. Adv. Phys. 24, 117-201 (1975).

69. Albertini, O. R. et al. Zone-center phonons of bulk, few-layer, and monolayer 1T$\mathrm{TaS}_{2}$ : detection of commensurate charge density wave phase through Raman scattering. Phys. Rev. B 93, 214109 (2016).

70. Samnakay, R. et al. Zone-folded phonons and the commensurate-incommensurate charge-density-wave transition in 1T-TaSe 2 thin films. Nano Lett. 15, 2965-2973 (2015).

71. Xi, X. et al. Strongly enhanced charge-density-wave order in monolayer $\mathrm{NbSe}_{2}$. Nat. Nanotechnol. 10, 765-769 (2015).

72. Lee, J.-U. et al. Ising-type magnetic ordering in atomically thin $\mathrm{FePS}_{3}$. Nano Lett. 16, 7433-7438 (2016).

73. Wang, $X$. et al. Raman spectroscopy of atomically thin two-dimensional magnetic iron phosphorus trisulfide $\left(\mathrm{FePS}_{3}\right.$ ) crystals. 2D Mater 3, 031009 (2016).

74. Kuo, C.-T. et al. Exfoliation and Raman spectroscopic fingerprint of few-layer $\mathrm{NiPS}_{3}$ van der Waals crystals. Sci. Rep. 6, 20904 (2016).

75. O'Hara, D. J. et al. Room temperature intrinsic ferromagnetism in epitaxial manganese selenide films in the monolayer limit. Nano Lett. 18, 3125-3131 (2018).

76. Bonilla, M. et al. Strong room-temperature ferromagnetism in $\mathrm{VSe}_{2}$ monolayers on van der Waals substrates. Nat. Nanotechnol. 13, 289 (2018).

77. Zhou, Y., Kanoda, K. \& Ng, T.-K. Quantum spin liquid states. Rev. Mod. Phys. 89, 025003 (2017).

78. Tian, Y., Gray, M. J., Ji, H., Cava, R. J. \& Burch, K. S. Magneto-elastic coupling in a potential ferromagnetic 2D atomic crystal. 2D Mater 3, 025035 (2016).

79. Knolle, J., Chern, G.-W., Kovrizhin, D., Moessner, R. \& Perkins, N. Raman scattering signatures of Kitaev spin liquids in $\mathrm{A}_{2} \mid \mathrm{IO}_{3}$ iridates with $\mathrm{A}=\mathrm{Na}$ or Li. Phys. Rev. Lett. 113, 187201 (2014).

80. Nasu, J., Knolle, J., Kovrizhin, D. L., Motome, Y. \& Moessner, R. Fermionic response from fractionalization in an insulating two-dimensional magnet. Nat. Phys. 12, 912 (2016).

81. Sandilands, L. J., Tian, Y., Plumb, K. W., Kim, Y.-J. \& Burch, K. S. Scattering continuum and possible fractionalized excitations in $\mathrm{a}-\mathrm{RuCl}_{3}$. Phys. Rev. Lett. 114, 147201 (2015).

82. McGuire, M. A. et al. High-temperature magnetostructural transition in van der Waals-layered a-MoCl . Phys. Rev. Mater. 1, 064001 (2017).

83. Pong, W.-T. \& Durkan, C. A review and outlook for an anomaly of scanning tunnelling microscopy (STM): superlattices on graphite. J. Phys. D 38, R329 (2005).

84. Eliel, G. et al. Intralayer and interlayer electron-phonon interactions in twisted graphene heterostructures. Nat. Commun. 9, 1221 (2018).

85. Carozo, V. et al. Raman signature of graphene superlattices. Nano Lett. 11, 4527-4534 (2011)

86. Jorio, A. \& Cançado, L. G. Raman spectroscopy of twisted bilayer graphene. Solid State Commun. 175, 3-12 (2013).

87. $\mathrm{Li}, \mathrm{H}$. et al. Thermal conductivity of twisted bilayer graphene. Nanoscale $\mathbf{6}$, 13402-13408 (2014).

88. Yu, H., Liu, G.-B., Tang, J., Xu, X. \& Yao, W. Moiré excitons: from programmable quantum emitter arrays to spin-orbit-coupled artificial lattices. Sci. Adv. 3, e1701696 (2017).
89. Alexeev, E. M. et al. Resonantly hybridized excitons in moir, superlattices in van der Waals heterostructures. Nature 567, 81 (2019).

90. Cong, C. \& Yu, T. Enhanced ultra-low-frequency interlayer shear modes in folded graphene layers. Nat. Commun. 5, 4709 (2014).

91. Eckmann, A. et al. Raman fingerprint of aligned graphene/h-BN superlattices. Nano Lett. 13, 5242-5246 (2013).

92. Kerrache, I., Julien, C. \& Sourisseau, C. Raman scattering studies of lithiumintercalated $\mathrm{NiPS}_{3}$. Solid State lonics 92, 37-43 (1996).

93. Beyerlein, R., Horowitz, H. \& Longo, J. The electrical properties of $A_{2}\left[R u_{2-x} A x\right] O_{7-y}$ $(\mathrm{A}=\mathrm{Pb}$ or $\mathrm{Bi})$ pyrochlores as a function of composition and temperature. J. Solid State Chem. 72, 2-13 (1988).

94. Zhang, X. et al. Raman spectroscopy of shear and layer breathing modes in multilayer MoS 2 . Phys. Rev. B 87, 115413 (2013)

95. Tang, T.-T. et al. A tunable phonon-exciton fano system in bilayer graphene. Nat. Nanotechnol. 5, 32 (2010).

96. Yang, J., Lee, J.-U. \& Cheong, H. Excitation energy dependence of Raman spectra of few-layer $\mathrm{WS}_{2}$. FlatChem 3, 64-70 (2017).

97. Liang, L., Puretzky, A. A., Sumpter, B. G. \& Meunier, V. Interlayer bond polarizability model for stacking-dependent low-frequency Raman scattering in layered materials. Nanoscale 9, 15340-15355 (2017).

98. Zhong, D. et al. Van der Waals engineering of ferromagnetic semiconductor heterostructures for spin and valleytronics. Sci. Adv. 3, e1603113 (2017).

99. Glamazda, A., Lemmens, P., Do, S.-H., Kwon, Y. S. \& Choi, K.-Y. Relation between Kitaev magnetism and structure in a-RuCl . Phys. Rev. B 95, 174429 (2017).

\section{ACKNOWLEDGEMENTS}

We acknowledge support from the National Key Research and Development Program of China (Grant No. 2016YFA0301204), the National Natural Science Foundation of China (Grant Nos.11874350 and 11434010) and CAS Key Research Program of Frontier Sciences (Grant No. ZDBS-LY-SLH004).

\section{AUTHOR CONTRIBUTIONS}

P.T. directed and supervised the project. X.C. drafted manuscript with the inputs from X.L. and M.L. All authors discussed and modified the manuscript at all stages.

\section{COMPETING INTERESTS}

The authors declare no competing interests.

\section{ADDITIONAL INFORMATION}

Correspondence and requests for materials should be addressed to P.-H.T.

Reprints and permission information is available at http://www.nature.com/ reprints

Publisher's note Springer Nature remains neutral with regard to jurisdictional claims in published maps and institutional affiliations.

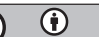

Open Access This article is licensed under a Creative Commons Attribution 4.0 International License, which permits use, sharing, adaptation, distribution and reproduction in any medium or format, as long as you give appropriate credit to the original author(s) and the source, provide a link to the Creative Commons license, and indicate if changes were made. The images or other third party material in this article are included in the article's Creative Commons license, unless indicated otherwise in a credit line to the material. If material is not included in the article's Creative Commons license and your intended use is not permitted by statutory regulation or exceeds the permitted use, you will need to obtain permission directly from the copyright holder. To view a copy of this license, visit http://creativecommons. org/licenses/by/4.0/.

(c) The Author(s) 2020 